\title{
AN ARITHMETIC THEORY OF ADJOINT PLANE CURVES
}

\section{BY \\ DANIEL GORENSTEIN}

Introduction. In classical algebraic geometry the adjoint curves to an irreducible plane curve are an essential tool in the study of the geometry on the curve. In this paper we shall give an algebro-arithmetic development of the theory of adjoint curves, and shall extend the classical results to irreducible plane curves with arbitrary singularities defined over arbitrary ground fields. Our definition of the adjoint condition at a given singular point of the curve is stated in terms of the conductor between the local ring of the point and its integral closure. The fundamental properties of the adjoint curves are then derived from corresponding properties of the conductor.

The single deepest and most important property of the adjoint curves is that, on a curve of order $m$, the adjoint curves of order $m-3$ cut out the complete canonical series. This property is equivalent to the fact that the degree of the fixed component of the adjoint series is twice the number of conditions which the adjoint curves impose on the curves of sufficiently high $\operatorname{order}\left({ }^{1}\right)$. We shall give two distinct and independent proofs of this proposition.

The first proof is a direct one, based upon a detailed analysis of the singularities of the given curve. This analysis, to which part I is devoted, applies equally well to algebraic number fields, and our treatment will include this case with that of algebraic function fields of one variable.

The second proof is more indirect, depending upon the Riemann-Roch theorem and a generalization of the classical representation theorem of the differentials of the first kind. This proof, which will be given in part II, holds only for plane curves whose function field is separably generated over the ground field.

\section{Part I. Local analysis of the SINGUlarities}

1. Algebraic preliminaries. In this section we shall develop the basic notation and results necessary for the local theory. The notation we establish will be valid simultaneously for algebraic number fields and algebraic function fields of one variable. Let $D_{0}$ be either the ring $I$ of ordinary integers or the

Received by the editors July 19, 1951.

(1) For example, an adjoint curve to a curve with ordinary multiple points $P_{1}, P_{2}, \cdots, P_{8}$ of multiplicities $r_{1}, r_{2}, \cdots, r_{s}$ respectively is one which has an $\left(r_{i}-1\right)$-fold point at each $P_{i}$. In this case, the degree of the fixed component is $\sum_{i=1}^{i} r_{i}\left(r_{i}-1\right)$, while the number of conditions which the adjoint curves impose on the curves of sufficiently high degree is $\sum_{i=1}^{:} r_{i}\left(r_{i}-1\right) / 2$. 
ring $k[X]$ of polynomials in an indeterminate $X$ with coefficients in an arbitrary field $k$. Let $F(Y) \in \mathrm{o}_{0}[Y]$ be irreducible and let $y$ be a root of $F(Y)$ $=0$. Call $\mathfrak{D}_{0}[Y], \mathfrak{D}_{0}[y]$ respectively $\mathfrak{D}$ and $\mathfrak{o}$. If $K$ denotes the quotient field of $\mathfrak{D}, K$ is an algebraic number field or an algebraic function field of one variable according as $\mathfrak{D}_{0}$ is $I$ or $k[X]$.

If $\mathfrak{p}$ is a maximal ideal in $\mathfrak{o}$, let $\mathfrak{o}_{\mathfrak{p}}$ be the ring of quotients of $\mathfrak{o}$ with respect to $\mathfrak{p}$. If $\mathfrak{p}_{0}=\mathfrak{p} \cap \mathfrak{D}_{0}$, denote by $\mathfrak{D}_{\mathfrak{p}_{0}}$ the quotient ring of $\mathfrak{D}_{0}$ with respect to $\mathfrak{p}_{0}$. $\mathfrak{D}_{\mathfrak{p}}$ and $\mathfrak{o}_{\mathfrak{p}_{0}}$ are local rings and, moreover, the completion $\mathfrak{D}_{\mathfrak{p}_{0}}^{*}$ of $\mathfrak{o}_{\mathfrak{p}_{0}}$ is a subring and subspace of the completion $\mathfrak{o}_{\mathfrak{p}}^{*}$ of $\mathrm{o}_{\mathfrak{p}}$.

It is well known that there exists at least one and at most a finite number of valuation rings $R_{i}$ of $K, i=1,2, \cdots, r$, which contain $o_{\mathfrak{p}}$ (compare Chevalley [2, Chapter I, Theorem 1 and Lemma 1] $\left({ }^{2}\right)$ ). If $\bar{D}_{\mathfrak{p}}$ denotes the integral closure of $\mathfrak{o}_{\mathfrak{p}}$ in $K$, it follows readily that $\overline{\mathfrak{D}}_{\mathfrak{p}}=\bigcap_{i=1}^{r} R_{i}$. Let $\mathfrak{m}_{i}$ be the ideal of nonunits in $R_{i}, i=1,2, \cdots, r$, and let $\mathfrak{m}_{i}^{\prime}=\mathfrak{m}_{i} \cap \overline{\mathfrak{o}}_{\mathrm{p}}$. Then $\mathfrak{m}_{i}^{\prime} \neq \mathfrak{m}_{j}^{\prime}$, $i \neq j, \mathfrak{m}_{i}^{\prime} \cap \mathfrak{o}_{\mathfrak{p}}=\mathfrak{p o p}_{\mathfrak{p}}$ for all $i, j=1,2, \cdots, r$, and the $\mathfrak{m}_{i}^{\prime}$ are the only ideals in $\overline{\mathfrak{D}}_{\mathfrak{p}}$ which contract to $\mathfrak{p o p}_{\mathfrak{p}}$ in $\mathfrak{o}_{\mathfrak{p}}$ (Zariski $[12$, p. 511]). It follows that $\mathfrak{m}_{1}^{\prime}, \mathfrak{m}_{2}^{\prime}, \cdots, \mathfrak{m}_{r}^{\prime}$ are the only ideals in $\overline{\mathfrak{o}}_{\mathfrak{p}}$ which are distinct from the zero or unit ideals, whence $\bar{D}_{\mathfrak{p}}$ is a semi-local ring in the sense of Chevalley. Since evidently $R_{i}$ is the ring of quotients of $\overline{\mathbf{D}}_{\mathfrak{p}}$ with respect to $\mathfrak{m}_{i}^{\prime}, i=1,2, \cdots, r$, Proposition 8 of Chevalley $[1$, p. 700$]$ implies that the completion $\overline{\mathfrak{D}}_{\mathfrak{p}}^{*}$ of the semi-local ring $\overline{0}_{\mathfrak{p}}$ is isomorphic to the direct sum of the completions $R_{i}^{*}$ of $R_{\boldsymbol{i}}$ :

$$
\overline{\mathrm{O}}_{\mathrm{p}}^{*} \cong \sum_{i=1}^{r} R_{i}^{*}
$$

On the other hand, let $\mathfrak{P}$ be the maximal ideal in $\mathfrak{D}$ whose residue $(\bmod F(Y))$ is $\mathfrak{p}$. Then $\mathfrak{o}_{\mathfrak{p}_{0}}^{*}$ can also be regarded as a subring and subspace of the completion $\mathfrak{D}_{\mathfrak{B}}^{*}$ of $\mathfrak{D}$ with respect to $\mathfrak{P}$. $\mathfrak{D}_{\mathfrak{B}}^{*}$ is a complete regular ring of dimension 2, and as such is a unique factorization domain (Cohen $[4$, Theorem 18]). The following relation exists between $\mathfrak{D}_{\mathfrak{B}}^{*}$ and $\mathfrak{o}_{\mathfrak{p}}^{*}$ (Chevalley $[1$, Proposition 5, p. 699]):

$$
\mathrm{D}_{\mathfrak{p}}^{*} \cong \mathfrak{D}_{\mathfrak{B}}^{*} / \mathfrak{D}_{\mathfrak{B}}^{*} F(Y)
$$

Let $F(Y)=\prod_{i=1}^{n} F_{i}^{*}$ be a factorization of $F(Y)$ into irreducible elements of $\mathfrak{D}_{\mathfrak{B}}^{*}$, in which each factor $F_{i}{ }^{*}$ is a nonunit in $\mathfrak{D}_{\mathfrak{B}}^{*}$. It is known that the zero ideal in $\mathrm{o}_{\mathrm{p}}^{*}$ is the intersection of prime ideals (Zariski $[13$, p. 356]). This implies, together with (2), that $F_{i}^{*} \neq F_{j}^{*}, i \neq j$. Define $\mathfrak{o}_{j}^{*}=\mathfrak{D}_{\mathfrak{B}}^{*} / F_{j}^{*}, j=1,2, \cdots$, $h$. Then each $\mathfrak{o}_{j}^{*}$ is itself a complete local domain. Moreover, the natural homomorphism of $\mathfrak{D}_{\mathfrak{B}}^{*}$ on $\mathfrak{D}_{j}{ }^{*}$ maps $\mathfrak{D}_{0}[Y]$ onto a subring of $\mathfrak{D}_{j}{ }^{*}$ which is isomorphic to $\mathfrak{o}$. Thus if $\Sigma_{j}^{*}$ denotes the quotient field of $\mathfrak{o}_{j}{ }^{*}$, we may identify $K$ with a subfield of $\Sigma_{j}^{*}, j=1,2, \cdots, h$. It can be further shown, by using

(2) Numbers in brackets refer to the bibliography at the end of the paper. 
Hensel's lemma (Cohen [4, Theorem 4]), that $\mathfrak{o}_{\mathfrak{p}}$ is then identified with a subring and subspace of $\mathfrak{o}_{j}^{*}$. Since the integral closure $\overline{\mathbf{b}}_{j}{ }^{*}$ of $\mathfrak{o}_{j}^{*}$ in $\Sigma_{j}^{*}$ is a complete discrete rank 1 valuation ring, its contraction $\overline{\boldsymbol{D}}_{j}^{*} \cap K$ is a valuation ring of $K$ containing $\mathfrak{D}_{\mathfrak{p}}$. It follows readily that each $\overline{\mathfrak{p}}_{j}{ }^{*}$ is isomorphic to one of the complete valuation rings $R_{i}^{*}$, and that distinct $\overline{\mathbf{b}}_{j}{ }^{*}$ correspond to distinct $R_{i}^{*}$. We therefore conclude that $h \leqq r$.

Conversely, $\hat{D}_{p}^{*}$ can be identified with a subring and subspace of $R_{i}^{*}$, $i=1,2, \cdots, r$. Since $F(Y)=0$ in $\mathfrak{o}_{\mathfrak{p}}$, this implies that some $F_{j}^{*}=0$ in $R_{i}^{*}$, whence it can be seen that $R_{i}^{*}$ is isomorphic to the corresponding complete valuation ring $\overline{\boldsymbol{D}}_{j}^{*}$. Thus $r=h$, and for a suitable ordering of the indices $R_{i}^{*}=\overline{\mathbf{b}}_{i}^{*}, i=1,2, \cdots, r$.

Let $\Sigma_{0}{ }^{*}$ be the quotient field of ${0_{p}}_{0}^{*}$. To simplify the exposition, we shall identify $K$ and $\Sigma_{0}{ }^{*}$ with their isomorphic images in $\Sigma_{i}^{*}$, shall identify $\overline{\boldsymbol{o}}_{i}{ }^{*}$ with $R_{i}^{*}, i=1,2, \cdots, r$, and shall identify $\overline{\mathfrak{o}}_{p}^{*}$ with $\sum_{i=1}^{r} R_{i}^{*}$. With this identification each $\Sigma_{i}{ }^{*}$ is a finite algebraic extension of $\Sigma_{0}{ }^{*}$. Since $F_{i}{ }^{*}$ and $F_{j}{ }^{*}$ are relatively prime in $\mathfrak{O}_{\mathfrak{B}}^{*}, i \neq j$, it can be further shown that $\mathfrak{D}_{\mathfrak{B}}^{*} / \mathfrak{O}_{\mathfrak{B}}^{*} F(Y)$ $\cong \sum_{i=1}^{r} \mathfrak{O}_{\mathfrak{B}}^{*} / \mathfrak{N}_{\mathfrak{B}}^{*} F_{i}^{*}$, whence $\mathfrak{o}_{\mathfrak{p}}^{*} \cong \sum_{i=1}^{r} \mathfrak{o}_{i}^{*}$ in view of $(2)$. We see then that $\mathfrak{0}_{p}^{*}$ is identified in a natural way with a subring and subspace of $\overline{\mathfrak{D}}_{p}^{*}$.

Let $v_{i}, v_{i}^{*}$ be the valuations whose corresponding valuation rings are respectively $R_{i}$ and $R_{i}^{*}, i=1,2, \cdots, r$. We shall say that $v_{i}$ or $v_{i}^{*}$ has center $p$ in $\mathbf{D}$.

Every element $z \in \mathfrak{o}_{p}{ }^{*}$ (or $\overline{\mathfrak{o}}_{p}^{*}$ ) has a unique representation of the form $z=\sum_{i=1}^{r} z_{i}, z_{i} \in R_{i}^{*}$. We define $v_{i}^{*}(z)=v_{i}^{*}\left(z_{i}\right), i=1,2, \cdots, r$. With this convention, we shall call $q^{*} C \mathfrak{o}_{p}^{*}$ (or $\overline{\mathfrak{o}}_{p}^{*}$ ) a valuation ideal or simply a $v_{i}^{*}$-ideal if $z \in \mathfrak{o}_{\mathfrak{p}}^{*}\left(\right.$ or $\left.\overline{\mathfrak{o}}_{\mathfrak{p}}^{*}\right), v_{i}^{*}(z) \geqq v_{i}^{*}\left(\mathfrak{q}^{*}\right)$, implies $z \in \mathfrak{q}^{*}$. We also agree to call the intersection of $v_{i}$-ideals, $i=1,2, \cdots, r$, in $\mathfrak{o}, \mathfrak{D}_{\mathfrak{p}}$, or $\overline{\mathfrak{D}}_{\mathfrak{p}}$, or the intersection of $v_{i}{ }^{*}$-ideals, $i=1,2, \cdots, r$, in $o_{p}^{*}$ or $\bar{o}_{p}{ }^{*}$ a complete $p$-ideal in its respective ring. In the one-one correspondence between the primary ideals in $\mathfrak{D}_{\mathfrak{p}}$ and $\mathfrak{p}_{\mathfrak{p}}^{*}$ the complete $p$-ideals correspond in a natural way: $q, q^{*}$ correspond if and only if $v_{i}^{*}\left(\mathfrak{q}^{*}\right)=v_{i}(\mathfrak{q}), i=1,2, \cdots, r$.

Let $k_{0}=\mathrm{D}_{0} / \mathfrak{p}_{0} ; k_{0}$ is either the prime field with $\mathfrak{p}_{0}$ elements or a finite extension of $k$. If $k(\mathfrak{p})=\mathfrak{o} / \mathfrak{p}, k(\mathfrak{p})$ is a finite extension of $k_{0}$. We define the degree $d_{\mathfrak{p}}$ of $\mathfrak{p}$ to be, on the one hand, the number of elements in $k(\mathfrak{p})$ or, on the other hand, $[k(\mathfrak{p}): k]$. Furthermore the residue field $\Delta_{i}$ of $R_{i}$ (or of $R_{i}^{*}$, since their residue fields are isomorphic) is in turn a finite extension of $k(p)$, $i=1,2, \cdots, r$. We define the degree $d_{i}$ of $v_{i}$ (or $\left.v_{i}^{*}\right)$ to be the number of elements in $\Delta_{i}$ or $\left[\Delta_{i}: k\right]$, as the case may be.

Let $q^{\prime} \subset q$ be zero-dimensional primary ideals in any one of the rings considered above (including $\mathfrak{D}$ and $\mathfrak{D}_{\mathfrak{p}}^{*}$ ). We allow $\mathfrak{q}=(1)$. The additive group of the ring $q / q^{\prime}$ can be regarded in a natural way as a finite-dimensional vector space over $k_{0}$, and in the algebraic function field case, a fortiori, as a vector space over $k$. We define then $\operatorname{dim} \mathfrak{q} / \mathfrak{q}^{\prime}$ to be the dimension of this vector space over $k$. In the alternative case, we define $\operatorname{dim} q / q^{\prime}$ to be the 
total number of elements in $\mathfrak{q} / \mathfrak{q}^{\prime}$. We also say a given set of elements of $\mathfrak{q}$ are linearly independent modulo $q^{\prime}$ if, on the one hand, their residues are linearly independent over $k$ or, on the other hand, are distinct elements of $\mathfrak{q} / \mathfrak{q}^{\prime}$.

The notion of the degree $d(\mathfrak{q})$ of a valuation ideal or complete $\mathfrak{p}$-ideal $\mathfrak{q}$ will be of considerable importance in the sequel. We define $d(\mathfrak{q})=d_{i} v_{i}(\mathfrak{q})$ or $d(\mathfrak{q})=\sum_{i=1}^{r} d_{i} \boldsymbol{v}_{i}(\mathfrak{q})$ according as $\mathfrak{q}$ is a $\boldsymbol{v}_{\boldsymbol{i}}$-ideal or complete $\mathfrak{p}$-ideal in $\mathfrak{D}, \mathfrak{o}_{\mathfrak{p}}$, or $\overline{\mathfrak{D}}_{\mathfrak{p}}$; and define $d(\mathfrak{q})=d_{i} v_{i}^{*}(\mathfrak{q})$ or $d(\mathfrak{q})=\sum_{i=1}^{r} d_{i} v_{i}^{*}(\mathfrak{q})$ according as $q$ is a $v_{i}{ }^{*}$-ideal or complete $\mathfrak{p}$-ideal in $\mathrm{o}_{p}^{*}, \overline{\mathrm{o}}_{\mathrm{p}}{ }^{*}, \mathrm{o}_{i}{ }^{*}$, or $R_{i}{ }^{*}$.

If $q^{\prime} \subset q$ are each $v_{i}$-ideals, $v_{i}^{*}$-ideals, or complete $p$-ideals, it is easily verified that $\operatorname{dim} \mathfrak{q} / \mathfrak{q}^{\prime} \leqq d\left(\mathfrak{q}^{\prime}\right)-d(\mathfrak{q})$. Moreover, it is a direct consequence of the independence of places that $\operatorname{dim} \mathfrak{q} / \mathfrak{q}^{\prime}=d\left(\mathfrak{q}^{\prime}\right)-d(\mathfrak{q})$ if $\mathfrak{q}, \mathfrak{q}^{\prime}$ are valuation ideals or complete $\mathfrak{p}$-ideals in any one of the integrally closed rings $\overline{\mathfrak{D}}_{\mathfrak{p}}, \overline{\mathbf{b}}_{\mathfrak{p}}{ }^{*}, R_{i}{ }^{*}$, and hence, in particular, that $d(\mathfrak{q}) \leqq \operatorname{dim}(1) / \mathfrak{q}$.

For valuation ideals or complete $\mathfrak{p}$-ideals in $\mathfrak{b}, \mathfrak{o}_{\mathfrak{p}}, \mathfrak{D}_{\mathfrak{p}}{ }^{*}$, or $\mathfrak{D}_{i}{ }^{*}$, we shall also introduce the integer $\delta(\mathfrak{q})=\operatorname{dim}(1) / \mathfrak{q}$. Thus $\delta(\mathfrak{q}) \leqq d(\mathfrak{q})$. We shall see in part II that this integer has a special geometric significance. If $q^{\prime} \subset q$ is also a $\boldsymbol{v}_{\boldsymbol{i}}$-ideal, $\boldsymbol{v}_{\boldsymbol{i}}{ }^{*}$-ideal, or complete $\mathfrak{p}$-ideal in $\mathfrak{D}, \mathfrak{o}_{\mathfrak{p}}, \mathfrak{D}_{\mathfrak{p}}{ }^{*}$, or $\mathfrak{D}_{i}{ }^{*}$, as the case may be, we have $\operatorname{dim} \mathfrak{q} / \mathfrak{q}^{\prime}=\operatorname{dim}(1) / \mathfrak{q}^{\prime}-\operatorname{dim}(1) / \mathfrak{q}$, whence

$$
\operatorname{dim} \mathfrak{q} / \mathfrak{q}^{\prime}=\delta\left(\mathfrak{q}^{\prime}\right)-\delta(\mathfrak{q}) \leqq d\left(\mathfrak{q}^{\prime}\right)-d(\mathfrak{q}) .
$$

This inequality leads finally to the concept of a regular ideal.

Definition. A $\boldsymbol{v}_{\boldsymbol{i}}$-ideal, $\boldsymbol{v}_{i}{ }^{*}$-ideal, or complete $\mathfrak{p}$-ideal $\mathfrak{q}$ in $\mathfrak{D}, \mathfrak{D}_{\mathfrak{p}}, \mathfrak{o}_{\mathfrak{p}}{ }^{*}$, or $\mathfrak{D}_{i}{ }^{*}$ is called a regular ideal if, for any $\boldsymbol{v}_{\boldsymbol{i}}$-ideal, $\boldsymbol{v}_{\boldsymbol{i}}{ }^{*}$-ideal, or complete $\mathfrak{p}$-ideal $\mathfrak{q}^{\prime} \subset \mathfrak{q}$, we have

$$
\delta\left(\mathfrak{q}^{\prime}\right)-\delta(\mathfrak{q})=d\left(\mathfrak{q}^{\prime}\right)-d(\mathfrak{q}) .
$$

\section{Some properties of the conductor.}

Theorem 1. If $\mathfrak{S}_{i}{ }^{*}$ is the conductor between $R_{i}^{*}$ and $\mathrm{o}_{i}{ }^{*}, i=1,2, \cdots, r$, then $\mathfrak{S}_{i}^{*}$ is the largest regular ideal in $\mathrm{o}_{i}{ }^{*}$.

Proof. To simplify the notation, we drop the subscript $i$. Let $z_{0} \in \complement^{*}$, $v^{*}\left(z_{0}\right)=v^{*}\left(\mathfrak{C}^{*}\right)$. If $z \in R^{*}$ and $v^{*}(z) \geqq v^{*}\left(\mathfrak{C}^{*}\right)$, then $v^{*}\left(z / z_{0}\right) \geqq 0$, whence $z / z_{0}$ $\in R^{*}$. Thus $z=z_{0}\left(z / z_{0}\right) \in \mathfrak{C}^{*}$, whence $\mathfrak{C}^{*}$ is in fact a $v^{*}$-ideal.

Let $\mathfrak{q}^{*} \subset \mathfrak{S}^{*}$ be a $v^{*}$-ideal, and let $a=v^{*}\left(\mathfrak{q}^{*}\right)-v^{*}\left(\mathfrak{S}^{*}\right)$. If $\mathfrak{S}^{*}$ is the ideal in $R^{*}$ of value $a, \operatorname{dim} R^{*} / \Im^{*}=d a$. If $\zeta_{j}, j=1,2, \cdots, d a$ form a basis for $R^{*}$ modulo $\mathfrak{S}^{*}$, the elements $z_{0} \zeta_{i}$ are in $\mathfrak{S}^{*}$ and are linearly independent modulo $q^{*}$, whence $\delta\left(q^{*}\right)-\delta\left(\mathfrak{\complement}^{*}\right) \geqq a d$, while $\delta\left(q^{*}\right)-\delta\left(\mathfrak{\complement}^{*}\right) \leqq a d$ by (3). We conclude that $\mathfrak{S}^{*}$ is a regular ideal in $\mathfrak{D}^{*}$.

We shall now show that for any regular ideal $\mathfrak{M}^{*}$ in $\mathfrak{D}^{*}, \mathfrak{M}^{*} \subset \mathfrak{C}^{*}$. It will suffice to prove:

$$
\text { z } E R^{*} \cdot v^{*}(z) \equiv v^{*}\left(M^{*}\right) \text { imply zE } 0^{*}
$$


For if $z \in R^{*}, \phi \in \mathfrak{M}^{*}, v^{*}(z \phi) \geqq v^{*}\left(\mathfrak{M}^{*}\right)$ whence $z \phi \in \mathfrak{o}^{*}$. Hence $\phi \in \mathfrak{S}^{*}$, and so $\mathfrak{M}^{*} \subset \mathfrak{C}^{*}$.

Let $\left.z \in R^{*}, v^{*}(z) \geqq v^{*}(\mathfrak{M})^{*}\right)$. Denote by $\left\{q_{j}^{*}\right\}$ the sequence of $v^{*}$-ideals in $\mathrm{D}^{*}$; and let $v^{*}(z)=b$. We construct a sequence of elements $z_{j} \in \mathfrak{q}_{b+j}^{*}, j=0,1,2$, ... such that $v^{*}\left(z-\sum_{j=0}^{h} z_{j}\right)>v^{*}\left(\mathfrak{q}_{b+h}^{*}\right)$ for every integer $h$. Suppose $z_{j}$, $j=0,1, \cdots, h-1$, have been constructed. Since $q_{b+h}^{*} \subset \mathfrak{M}^{*}, \mathfrak{q}_{b+h}^{*}$ is itself a regular ideal, and so $\delta\left(\mathfrak{q}_{b+h+1}^{*}\right)-\delta\left(\mathfrak{q}_{b+h}^{*}\right)=d$. Let $t \in R^{*}$ with $v^{*}(t)=v^{*}\left(q_{b+h}^{*}\right)$. The previous equality implies at once that for any $\alpha \in \Delta$, there exists an element $\eta \in \mathfrak{q}_{b+h}^{*}$ such that the residue in $\Delta$ of $\eta / t$ is $\alpha$. It follows from this remark that we can find an element $z_{h} \in \mathrm{q}_{b+h}^{*}$ such that $v^{*}\left(\left[z-\sum_{j=0}^{h-1} z_{j}\right]-z_{h}\right)>v^{*}(t)$, whence $v^{*}\left(z-\sum_{j=0}^{h} z_{j}\right)>v^{*}\left(\mathfrak{q}_{b+h}^{*}\right)$, as asserted.

Since $\bigcap_{j=0}^{\infty} \mathfrak{q}_{j}^{*}=(0)$ and since $\mathfrak{o}^{*}$ is complete, it follows from Chevalley $\left[1\right.$, Lemma 7, p. 695] that the series $\sum_{j=0}^{\infty} z_{j}$ is convergent in $0^{*}$ to an element $z^{*}$. Since by construction $v^{*}\left(z-z^{*}\right)=\infty, z-z^{*}=0$, and hence $z \in 0^{*}$, which proves (5).

On the basis of the preceding analysis, we prove two theorems concerning the conductor $\mathfrak{S}_{p}$ between $\overline{\mathfrak{D}}_{\mathfrak{p}}$ and $\mathfrak{o}_{\mathfrak{p}}$. The first theorem gives the complete structure of $\mathbb{E}_{p}$, while the second gives a valuation-theoretic characterization of $\mathfrak{E}_{p}$.

TheOREM 2. Let $\mathfrak{G}_{p}^{*}$ be the conductor between $\overline{\mathrm{D}}_{\mathrm{p}}{ }^{*}$ and $\mathrm{o}_{\mathrm{p}}{ }^{*}$, and let $\omega_{i}^{*}$ be the residue in $\mathrm{D}_{i}^{*}$ of the elements $\prod_{j \neq i} F_{j}^{*}, i=1,2, \cdots, r$. Then

$$
\mathfrak{C}_{\mathfrak{p}}^{*}=\sum_{i=1}^{r} \mathfrak{C}_{i}^{*} \omega_{i}^{*}, \quad \mathfrak{C}_{\mathfrak{p}}=\mathfrak{C}_{\mathfrak{p}}^{*} \cap \mathfrak{D}_{\mathfrak{p}}, \quad \text { and } \quad \mathfrak{C}_{\mathfrak{p}}^{*}=\mathfrak{D}_{\mathfrak{p}}^{*} \mathfrak{C}_{\mathfrak{p}} \text {. }
$$

TheOREM 3. $\mathfrak{G}_{\mathfrak{p}}$ is the largest regular $\mathfrak{p}$-ideal in $\mathrm{D}_{\mathfrak{p}}$.

We prove both theorems simultaneously. Let $\Omega_{i}^{*}=\prod_{j \neq 1} F_{j}^{*}, i$ $=1,2, \cdots, r$, and let $\mathfrak{O}^{*}$ be the ideal $\left(\Omega_{1}^{*}, \Omega_{2}^{*}, \cdots, \Omega_{r}^{*}\right)$ in $\mathfrak{D}_{\mathfrak{B}}^{*}$. Since $\mathfrak{D}_{\mathfrak{P}}^{*} F(Y)=\bigcap_{i=1}^{r} \mathfrak{D}_{\mathfrak{B}}^{*} \Omega_{i}^{*}$, it is easily verified that $\mathfrak{D}^{*} / \mathfrak{D}_{\mathfrak{P}}^{*} F(Y)=\sum_{i=1}^{r} \mathfrak{D}_{i}^{*} \omega_{i}^{*}$. In view of the identification of ${D_{p}}^{*}$ with a subring of $\overline{\mathfrak{D}}_{p}^{*}$, it follows that $\sum_{i=1}^{r} \mathfrak{D}_{i}^{*} \omega_{i}^{*} \subset \mathfrak{D}_{p}^{*}$ and hence also $\sum_{i=1}^{r} \mathcal{C}_{i}^{*} \omega_{i}^{*} \subset \mathfrak{D}_{p}^{*}$. The first assertion of Theorem 2 thus has a meaning. In addition, it follows that $\overline{\mathfrak{S}}^{*}=\sum_{i=1}^{r} \mathfrak{C}_{i}^{*} \omega_{i}^{*}$ is an ideal in $\mathfrak{D}_{p}^{*}$ and, since $\overline{\mathfrak{C}}^{*}$ is manifestly the conductor between $\overline{\mathfrak{D}}_{p}{ }^{*}$ and $\sum_{i=1}^{r} 0_{i}^{*} \omega_{i}^{*}$, that $\overline{\mathfrak{C}}^{*} \subset \mathfrak{C}_{\mathfrak{p}}^{*}$. The proof that $\mathfrak{C}_{\mathfrak{p}}^{*} \subset \overline{\mathfrak{C}}^{*}$ depends upon two assertions:

(a) $\overline{\mathfrak{C}}^{*}$ is a regular ideal.

(b) No ideal containing $\overline{\mathfrak{C}}^{*}$ properly is a regular ideal.

Let $\mathfrak{q}^{*} \subset \overline{\mathfrak{C}}^{*}$ be a complete $\mathfrak{p}$-ideal in $\mathfrak{o}_{\mathfrak{p}}^{*}$. Denote by $\mathfrak{D}_{i}^{*}, i=1,2, \cdots, r$, the $v_{i}^{*}$-ideal in $\mathfrak{D}_{i}^{*}$ such that $v_{i}^{*}\left(\mathfrak{Q}_{i}^{*} \omega_{i}^{*}\right)=v_{i}^{*}\left(\mathfrak{q}^{*}\right)$. If $z \in \mathfrak{q}^{*}$, write $z=\sum_{i=1}^{r} z_{i}$, $z_{i} \in R_{i}^{*}$. If $\phi_{i}=z_{i} / \omega_{i}^{*}, i=1,2, \cdots, r, v_{i}^{*}\left(\phi_{i}\right) \geqq v_{i}^{*}\left(\mathfrak{D}_{i}^{*}\right) \geqq v_{i}^{*}\left(\mathfrak{S}_{i}^{*}\right)$, whence $\phi_{i}$ $\in \mathrm{o}_{i}{ }^{*}$, and, in fact, $\phi_{i} \in \mathfrak{Q}_{i}{ }^{*}$. Thus $z=\sum_{i=1}^{r} \phi_{i} \omega_{i}^{*}$, and it follows that $q^{*}$ $=\sum_{i=1}^{r} \mathfrak{Q}_{i}^{*} \omega_{i}^{*}$. In particular, this implies that $\overline{\mathfrak{C}}^{*}$ is a complete $\mathfrak{p}$-ideal.

Since, by Theorem 1 , each $\mathfrak{C}_{i}^{*}$ is regular, 


$$
\delta\left(\mathfrak{Q}_{i}^{*}\right)-\delta\left(\mathfrak{S}_{i}^{*}\right)=d_{i}\left[v_{i}^{*}\left(\mathfrak{Q}_{i}^{*}\right)-v_{i}^{*}\left(\mathfrak{G}_{i}^{*}\right)\right], \quad i=1,2, \cdots, r,
$$

whence $\delta\left(\mathfrak{q}^{*}\right)-\delta\left(\overline{\mathfrak{S}}^{*}\right)=\sum_{i=1}^{r} d_{i}\left[v_{i}^{*}\left(\mathfrak{Q}_{i}^{*}\right)-v_{i}^{*}\left(\mathfrak{C}_{i}^{*}\right)\right]=d\left(\mathfrak{q}^{*}\right)-d\left(\overline{\mathfrak{C}}^{*}\right)$. Thus $\overline{\mathfrak{S}}^{*}$ is a regular ideal as asserted in (a).

Consider now the complete ideals $\mathfrak{A}_{j}^{*}$ in $\mathfrak{o}_{\mathfrak{p}}^{*}$ defined by the relations:

$$
\boldsymbol{v}_{j}^{*}\left(\mathfrak{A}_{j}^{*}\right)=\boldsymbol{v}_{j}^{*}\left(\overline{\mathfrak{\complement}}^{*}\right)-1, \quad \boldsymbol{v}_{k}^{*}\left(\mathfrak{A}_{j}^{*}\right)=\boldsymbol{v}_{k}^{*}\left(\overline{\mathfrak{\complement}}^{*}\right), \quad k \neq j, j=1,2, \cdots, \boldsymbol{r} .
$$

If $\mathfrak{q}^{*} \supset \overline{\mathfrak{C}}^{*}$ properly is a complete $\mathfrak{p}$-ideal in $\mathfrak{D}_{\mathfrak{p}}^{*}, \mathfrak{q}^{*} \supset \mathfrak{A}_{j}^{*}$ for some $j$. To prove that $q^{*}$ is not a regular ideal, it is sufficient, in view of (3), to show that

$$
\delta\left(\overline{\mathfrak{C}}^{*}\right)-\delta\left(\mathfrak{A}_{j}^{*}\right)<d_{j}, \quad j=1,2, \cdots, r .
$$

For definiteness, take $j=1$, and suppose $z \in \mathfrak{P}_{1}^{*}$. Write $z=\sum_{i=1}^{r} z_{i}, z_{i} \in R_{i}^{*}$. By assumption, $v_{i}^{*}\left(z_{i}\right) \geqq v_{i}^{*}\left(\widehat{\complement}^{*}\right)=v_{i}^{*}\left(\mathfrak{C}_{i}^{*} \omega_{i}^{*}\right)$, for all $i>1$, whence $z_{i} \in \mathfrak{C}_{i}^{*} \omega_{i}^{*}$, $i>1$. It follows that we can find an element $z^{\prime} \in \bar{\complement}^{*}$ such that $z-z^{\prime}=z_{1}$. If $Z, Z^{\prime}$ are representatives of $z, z^{\prime}$ in $\mathfrak{D}_{\mathfrak{B}}^{*}$, this implies that $Z-Z^{\prime}=\Phi \Omega_{1}^{*}$, $\Phi \in D_{\mathfrak{B}}^{*}$. If $\phi$ is the residue of $\Phi$ in $\mathfrak{o}_{\mathfrak{p}}^{*}$, we conclude that $z \equiv \phi \omega_{1}^{*}\left(\bmod \overline{\mathfrak{S}}^{*}\right)$. This implies in turn that $\operatorname{dim} \mathfrak{A}_{1}^{*} / \bar{\complement}^{*} \leqq \operatorname{dim} \mathfrak{q}_{1}^{*} / \mathfrak{S}_{1}^{*}$, where $\mathfrak{q}_{1}^{*}$ is the $v_{1}^{*}$-ideal in $\mathfrak{o}_{1}^{*}$ immediately preceding $\mathfrak{S}_{1}^{*}$. By Theorem 1 , dim $\mathfrak{q}_{1}^{*} / \mathfrak{S}_{1}^{*}=\delta\left(\mathfrak{S}_{1}^{*}\right)-\delta\left(\mathfrak{q}_{1}^{*}\right)<d_{1}$. This proves (6) and hence also (b).

Suppose now $z \in \mathfrak{G}_{p}^{*}$ and $z \in \bar{\complement}^{*}$. Then for some $i$, say $i=1, v_{1}^{*}(z)<v_{1}^{*}\left(\bar{\complement}^{*}\right)$. Define the complete $\mathfrak{p}$-ideals $\mathfrak{B}_{1}^{*}, \mathfrak{D}_{1}^{*}$ in $\overline{\mathfrak{D}}_{\mathfrak{p}}{ }^{*}$, as follows:

$$
\begin{aligned}
& v_{1}^{*}\left(\mathfrak{B}_{1}^{*}\right)=v_{1}^{*}\left(\overline{\mathfrak{C}}^{*}\right)-v_{1}^{*}(z)-1, \quad v_{i}^{*}\left(\mathfrak{B}_{1}^{*}\right)=v_{i}^{*}\left(\overline{\mathfrak{S}}^{*}\right), \quad i>1 ; \\
& v_{1}^{*}\left(\mathfrak{D}_{1}^{*}\right)=v_{1}^{*}\left(\overline{\mathfrak{C}}^{*}\right)-v_{1}^{*}(z), \quad v_{i}^{*}\left(\mathfrak{D}_{1}^{*}\right)=v_{i}^{*}\left(\overline{\mathfrak{S}}^{*}\right), \quad i>1 .
\end{aligned}
$$

There exist $d_{1}$ elements $\psi_{i}, i=1,2, \cdots, d_{1}$, in $\mathfrak{B}_{1}^{*}$ linearly independent modulo $\mathfrak{D}_{1}^{*}$. The $d_{1}$ elements $z \psi_{i}, i=1,2, \cdots, d_{1}$, are then in $\mathfrak{A}_{1}^{*}$ and are linearly independent modulo $\widetilde{\mathfrak{S}}^{*}$. Thus $\delta\left(\overline{\mathfrak{C}}^{*}\right)-\delta\left(\mathfrak{P}_{1}^{*}\right)=d_{1}$, in contradiction to (6). Therefore $\mathfrak{E}_{\mathbb{p}}^{*} \subset \overline{\mathfrak{C}}^{*}$, which proves the first assertion of Theorem 2 .

It follows from the one-one correspondence between the complete ideals in $\mathfrak{o}_{p}{ }^{*}$ and $\mathfrak{o}_{\mathfrak{p}}$ that $\mathfrak{G}_{p}{ }^{*} \cap \mathfrak{o}_{\mathfrak{p}}$ is a regular ideal, while for each $i=1,2, \cdots, r$, $\mathfrak{A}_{i}^{*} \cap \mathfrak{D}_{\mathfrak{p}}$ is not a regular ideal, and also that $\mathfrak{S}_{\mathfrak{p}}^{*}=\mathfrak{D}_{\mathfrak{p}}{ }^{*}\left(\mathfrak{C}_{\mathfrak{p}}^{*} \cap \mathfrak{D}_{\mathfrak{p}}\right)$. The argument which proved that $\mathfrak{C}_{p}^{*} \subset \overline{\mathfrak{C}}^{*}$ can now be repeated to show that $\mathfrak{S}_{p} \subset \mathbb{C}_{p}^{*} \cap \mathfrak{o}_{p}$.

Conversely, if $z \in \mathfrak{C}_{\mathfrak{p}}^{*} \cap \mathfrak{o}_{\mathfrak{p}}, \quad \eta \in \overline{\mathfrak{o}}_{\mathfrak{p}}, \quad z \eta \in \mathfrak{o}_{\mathfrak{p}}^{*} \cap \overline{\mathfrak{o}}_{\mathfrak{p}}=\mathfrak{o}_{\mathfrak{p}}$, whence $\mathfrak{S}_{\mathfrak{p}}^{*} \cap \mathfrak{o}_{\mathfrak{p}} \subset \mathfrak{C}_{\mathfrak{p}}$. Hence $\mathfrak{S}_{\mathfrak{p}}^{*} \cap \mathfrak{D}_{\mathfrak{p}}=\mathfrak{S}_{\mathfrak{p}}$, which completes the proof of Theorem 2 .

To complete the proof of Theorem 3 , it remains to show that every regular 1deal in $\mathfrak{o}_{\mathfrak{p}}$ is contained in $\mathfrak{G}_{\mathfrak{p}}$. It will suffice to prove that if $\mathfrak{M}^{*}$ is a regular ideal in $\mathfrak{o}_{\mathfrak{p}}^{*}$, then $\mathfrak{M}^{*} \subset \mathfrak{G}_{p}^{*}$. Suppose $z \in \mathfrak{o}_{\mathfrak{p}}{ }^{*}$ and $v_{i}^{*}(z) \geqq v_{i}^{*}\left(\mathfrak{M}^{*}\right)$. Define an ordered sequence $\left\{\mathfrak{q}_{m}^{*}\right\}$ of complete $p$-ideals in $\mathfrak{o}_{p}^{*}$ by the relations: $v_{i}^{*}\left(\mathfrak{q}_{m}^{*}\right)$ $=v_{i}^{*}(z)+m, i=1,2, \cdots, r$. Since $\mathfrak{q}_{m}{ }^{*} \subset \mathfrak{M}^{*}, \mathfrak{q}_{m}^{*}$ is a regular ideal, $m$ $=0,1,2, \cdots, r$. It follows as in the proof of Theorem 1 that we can construct elements $z_{m} \in \mathfrak{q}_{m}{ }^{*}, m=0,1,2, \cdots$, such that, for each integer $h$, $v_{i}^{*}\left(z-\sum_{m=0}^{h} z_{m}\right)>v_{i}^{*}\left(\mathfrak{q}_{m+h}^{*}\right), i=1,2, \cdots, r$. Since $\mathfrak{q}_{m+1}^{*} \subset \mathfrak{q}_{m}^{*}, \bigcap_{m=0}^{\infty} \mathfrak{q}_{m}^{*}=(0)$, and $\mathfrak{o}_{\mathfrak{p}}{ }^{*}$ is complete, it follows again from Lemma 7 , Chevalley $[1$, p. 695], 
that the series $\sum_{m=0}^{\infty} z_{m}$ converges to an element of $\mathfrak{o}_{\mathfrak{p}}^{*}$, and hence that $z \in \mathfrak{o}_{\mathfrak{p}}^{*}$. We conclude at once that $\mathfrak{M}^{*} \subset \mathfrak{C}_{p}^{*}$. Q.E.D.

3. Semi-groups of positive integers. In order to establish a deeper property of the conductor, we must first consider certain arithmetic properties of integers which can be expressed in a specially designated way as a linear combination of $n$ positive integers $b_{1}, b_{2}, \cdots, b_{n}$.

Let $\kappa_{i}=\left(b_{1}, b_{2}, \cdots, b_{i}\right), i=1,2, \cdots, n$, and let $s_{2}, s_{3}, \cdots, s_{n}$ be another set of positive integers. We shall be interested in linear combinations of $b_{1}, b_{2}, \cdots, b_{n}$ with non-negative coefficients which for $i=2,3, \cdots, n$ are respectively $<s_{i}\left(\kappa_{i-1} / \kappa_{i}\right)$. An integer which can be written in this fashion will be called a proper combination of $b_{1}, b_{2}, \cdots, b_{n}$. We denote by $R_{m}\left(b_{1}, b_{2}, \cdots, b_{n}\right)$ the set of integers which can be expressed in exactly $m$ distinct proper combinations of $b_{1}, b_{2}, \cdots, b_{n}$. For any integer $c$, if $c \in R_{m}\left(b_{1}, b_{2}, \cdots, b_{n}\right)$, we shall write $\chi(c)=m$.

We shall also have to consider linear combinations of $b_{1}, b_{2}, \cdots, b_{n}$ with positive coefficients which for $i=2,3, \cdots, n$ are respectively $\leqq s_{i}\left(\kappa_{i-1} / \kappa_{i}\right)$. Such a linear combination of $b_{1}, b_{2}, \cdots, b_{n}$ will be called proper ${ }^{+}$, and $R_{m}^{+}\left(b_{1}, b_{2}, \cdots, b_{n}\right)$ will denote the set of integers expressible in exactly $m$ distinct proper ${ }^{+}$combinations of $b_{1}, b_{2}, \cdots, b_{n}$.

Let $d=\prod_{i=2}^{n} s_{i}$. We define two integers $\mu_{d}\left(b_{1}, b_{2}, \cdots, b_{n}\right), \nu_{d}\left(b_{1}, b_{2}, \cdots, b_{n}\right)$ which will be important in our considerations: $\mu_{d}\left(b_{1}, b_{2}, \cdots, b_{n}\right)$ $=\sum_{i=2}^{n} s_{i}\left(\kappa_{i-1} / \kappa_{i}\right) b_{i}, \nu_{d}\left(b_{1}, b_{2}, \cdots, b_{n}\right)=\mu_{d}\left(b_{1}, b_{2}, \cdots, b_{n}\right)+1-\sum_{i=1}^{n} b_{i}$.

Theorem 4. Assume $\left(b_{1}, \cdots, b_{n}\right)=1$. Then

(a) if $c \geqq \nu_{d}\left(b_{1}, \cdots, b_{n}\right), c \in R_{d}\left(b_{1}, \cdots, b_{n}\right)$;

(b) $\nu_{d}\left(b_{1}, \cdots, b_{n}\right)-1 \in R_{d-1}\left(b_{1}, \cdots, b_{n}\right)$;

(c) $\sum_{c=0}^{\nu_{d}\left(b_{1}, \cdots, b_{n}\right)-1} \chi(c)=2^{-1} d \cdot \nu_{d}\left(b_{1}, \cdots, b_{n}\right)$.

Proof. We establish a sequence of shorter propositions which together will form the proof of the theorem.

(d) If $c>\mu_{d}\left(b_{1}, b_{2}, \cdots, b_{n}\right)$, then $c \in R_{d}^{+}\left(b_{1}, \cdots, b_{n}\right)$.

If $n=2,\left(b_{1}, b_{2}\right)=1, s_{2}=d$. The relation $c-x_{2} b_{2} \equiv c-x_{2}^{\prime} b_{2}\left(\bmod b_{1}\right)$ implies $x_{2} \equiv x_{2}^{\prime}\left(\bmod b_{1}\right)$. It follows that $c-z_{2} b_{2} \equiv 0\left(\bmod b_{1}\right)$ for some integer $z_{2}$ such that $1 \leqq z_{2} \leqq b_{1}$. If $c>\mu_{d}\left(b_{1}, b_{2}\right)=d b_{1} b_{2}$, it follows that $c-\left(z_{2}+r b_{1}\right) b_{2}$ is a positive multiple of $b_{1}$ for $r=0,1,2, \cdots, d-1$. On the other hand, if $c=y_{1} b_{1}+y_{2} b_{2}$ is a proper combination of $b_{1}, b_{2}, y_{2}=z_{2}+r b_{1}$ for some $r=0,1, \cdots, d-1$. Hence $c \in R_{d}{ }^{+}\left(b_{1}, b_{2}\right)$.

We proceed by induction. Since $\left(b_{1} / \kappa_{n-1}, \cdots, b_{n-1} / \kappa_{n-1}\right)=1$, we can apply induction to these $n-1$ integers. Observe also that $s_{i}\left(\kappa_{i-1} / \kappa_{n-1}\right) /\left(\kappa_{i} / \kappa_{n-1}\right)$ $=s_{i}\left(\kappa_{i-1} / \kappa_{i}\right), i=2,3, \cdots, n-1$, so that the upper bounds on the coefficients of $b_{2} / \kappa_{n-1}, \cdots, b_{n-1} / \kappa_{n-1}$ are the same as those of $b_{2}, \cdots, b_{n-1}$.

Let $d^{\prime}=\prod_{i=2}^{n-1} s_{i}$, so that $d=d^{\prime} s_{n}$. Since $\left(\kappa_{n-1}, b_{n}\right)=1, c-\left(z_{n}+r \kappa_{n-1}\right) b_{n}$ $\equiv 0\left(\bmod \kappa_{n-1}\right)$ for some $z_{n}$ such that $1 \leqq z_{n} \leqq \kappa_{n-1}$ and for $r=0,1, \cdots, s_{n}-1$. Let $c_{r}=\left(c-\left(z_{n}+r \kappa_{n-1}\right) b_{n}\right) / \kappa_{n-1}, r=0,1, \cdots, s_{n}-1$. Now, by definition, 
$\mu_{d}\left(b_{1}, b_{2}, \cdots, b_{n}\right)=\kappa_{n-1} \mu_{d^{\prime}}\left(b_{1} / \kappa_{n-1}, \cdots, b_{n-1} / \kappa_{n-1}\right)+s_{n} \kappa_{n-1} b_{n}$. It follows from this equality that each $c_{r}>\mu_{d^{\prime}}\left(b_{1} / \kappa_{n-1}, \cdots, b_{n-1} / \kappa_{n-1}\right)$, since $c>\mu_{d}\left(b_{1}, b_{2}, \cdots\right.$, $\left.b_{n}\right)$. Hence by induction $c_{r} \in R_{d^{\prime}}\left(b_{1} / \kappa_{n-1}, \cdots, b_{n-1} / \kappa_{n-1}\right), \quad r=0,1, \cdots$, $s_{n}-1$. Thus $c \in R_{a}\left(b_{1}, b_{2}, \cdots, b_{n}\right)$ with $a \geqq s_{n} d^{\prime}=d$. On the other hand, if $c-y_{n} b_{n} \equiv 0\left(\bmod \kappa_{n-1}\right)$ with $0<y_{n} \leqq s_{n} \kappa_{n-1}, y_{n}=z_{n}+r \kappa_{n-1}$ for some $r=0,1, \cdots$, $s_{n}-1$. We conclude that $c \in R_{d}^{+}\left(b_{1}, b_{2}, \cdots, b_{n}\right)$.

(e) If $c \geqq \nu_{d}\left(b_{1}, b_{2}, \cdots, b_{n}\right)$, then $c \in R_{d}\left(b_{1}, b_{2}, \cdots, b_{n}\right)$.

In fact, if $c \geqq \nu_{d}\left(b_{1}, b_{2}, \cdots, b_{n}\right), c+\sum_{i=1}^{n} b_{i}>\mu_{d}\left(b_{1}, b_{2}, \cdots, b_{n}\right)$, whence, by (d), $c+\sum_{i=1}^{n} b_{i} \in R_{d}^{+}\left(b_{1}, b_{2}, \cdots, b_{n}\right)$. Thus $c \in R_{d}\left(b_{1}, b_{2}, \cdots, b_{n}\right)$.

(f) $\mu_{d}\left(b_{1}, b_{2}, \cdots, b_{n}\right) \in R_{d-1}^{+}\left(b_{1}, b_{2}, \cdots, b_{n}\right)$.

For the case $n=2, \mu_{d}\left(b_{1}, b_{2}\right)=d b_{1} b_{2}$. The representations of $d b_{1} b_{2}$ are all of the form $d b_{1} b_{2}=\left(d b_{2}-r b_{2}\right) b_{1}+\left(r b_{1}\right) b_{2}$, whence the proper ${ }^{+}$representations of $d b_{1} b_{2}$ occur for $r=1,2, \cdots, d-1$.

We use induction once again. Since $\mu_{d}\left(b_{1}, b_{2}, \cdots, b_{n}\right) \equiv 0\left(\bmod \kappa_{n-1}\right)$ and since $\left(\kappa_{n-1}, b_{n}\right)=1$, every proper ${ }^{+}$representation of $\mu_{d}\left(b_{1}, b_{2}, \cdots, b_{n}\right)$ is of the form $\sum_{i=1}^{n-1} z_{i} b_{i}+r \kappa_{n-1} b_{n}, r=1,2, \cdots, s_{n}$. Furthermore $e_{r}$ $=\left(\mu_{d}\left(b_{1}, \cdots, b_{n}\right)-r \kappa_{n-1} b_{n}\right) / \kappa_{n-1}=\mu_{d^{\prime}}\left(b_{1} / \kappa_{n-1}, \cdots, b_{n-1} / \kappa_{n-1}\right)+\left(s_{n}-r\right) b_{n}$, $r=1,2, \cdots, s_{n}$. By (d), $e_{i}=R_{d^{\prime}}^{+}\left(b_{1}, b_{2}, \cdots, b_{n}\right), i=1,2, \cdots, s_{n}-1$. By our induction hypothesis $e_{s_{n}} \in R_{d,-1}^{+}\left(b_{1} / \kappa_{n-1}, \cdots, b_{n-1} / \kappa_{n-1}\right)$. Consequently $\mu_{d}\left(b_{1}, b_{2}, \cdots, b_{n}\right) \in R_{d-1}^{+}\left(b_{1}, b_{2}, \cdots, b_{n}\right)$.

(g) $\nu_{d}\left(b_{1}, b_{2}, \cdots, b_{n}\right)-1 \in R_{d-1}\left(b_{1}, b_{2}, \cdots, b_{n}\right)$.

This follows at once from (f) and the fact that $\mu_{d}\left(b_{1}, b_{2}, \cdots, b_{n}\right)$ $=\nu_{d}\left(b_{1}, b_{2}, \cdots, b_{n}\right)-1+\sum_{i=1}^{n} b_{i}$.

(h) For any $c$, if $c \in R_{a}\left(b_{1}, b_{2}, \cdots, b_{n}\right)$, then $a \leqq d$.

The statement: for any $m$, if $m \in R_{a}^{+}\left(b_{1}, b_{2}, \cdots, b_{n}\right)$, then $a \leqq d$, is proved in the same manner as (d). Applying this remark to $m=c+\sum_{i=1}^{n} b_{i}$ will prove $(\mathrm{h})$.

(i) If $c \leqq \nu_{d}\left(b_{1}, b_{2}, \cdots, b_{n}\right)-1$ and $c \in R_{a}\left(b_{1}, b_{2}, \cdots, b_{n}\right)$, then $\nu_{d}\left(b_{1}, b_{2}, \cdots, b_{n}\right)-1-c \in R_{d-a}\left(b_{1}, b_{2}, \cdots, b_{n}\right)$.

Consider first the case $n=2$. If $c \in R_{a}\left(b_{1}, b_{2}\right)$, we can find an integer $z_{2}$, $0 \leqq z_{2}<b_{1}$, such that $c=z_{1} b_{1}+z_{2} b_{2}$. All the representations of $c$ are then of the form $c=\left(z_{1}-r b_{2}\right) b_{1}+\left(z_{2}+r b_{1}\right) b_{2}$, and the proper representations occur for $r=0,1, \cdots, a-1$. Hence $(a-1) b_{2} \leqq z_{1}<a b_{2}$. Since $\nu_{d}\left(b_{1}, b_{2}\right)-1$ $=\left[(d-1) b_{2}-1\right] b_{1}+\left(b_{1}-1\right) b_{2}$, it follows that $\nu_{d}-1-c=y_{1} b_{1}+y_{2} b_{2}$ with $y_{1}=(d-1) b_{2}-1-z_{1}$, whence $(d-1-a) b_{2} \leqq y_{1}<(d-a) b_{2}$, and with $y_{2}=b_{1}-1$ $-z_{2}$, whence $0 \leqq y_{2}<b_{1}-1$. We conclude that $\left(y_{1}-r b_{2}\right) b_{1}+\left(y_{2}+r b_{1}\right) b_{2}$ are proper representations of $\nu_{d}-1-c$ if and only if $r=0,1, \cdots, d-a-1$. Hence $\nu_{d}-1-c \in R_{d-a}\left(b_{1}, b_{2}\right)$.

We proceed by induction. Assume $c \in R_{a}\left(b_{1}, b_{2}, \cdots, b_{n}\right)$. If $c-z_{n} b_{n}$ $\equiv 0\left(\bmod \kappa_{n-1}\right)$ with $0 \leqq z_{n}<\kappa_{n-1}$, let, as above, $c_{r}=\left(c-\left(z_{n}+r \kappa_{n-1}\right) b_{n}\right) / \kappa_{n-1}$, $r=0,1, \cdots, s_{n}-1$. It is clear that distinct representations of $c_{r}$ as a proper combination of $b_{1} / \kappa_{n-1}, \cdots, b_{n-1} / \kappa_{n-1}$ will yield distinct representations of $c$ as a proper combination of $b_{1}, b_{2}, \cdots, b_{n}$, and will, in fact, give all possible 
proper representations of $c$. If then $c_{r} \in R_{a(r)}\left(b_{1} / \kappa_{n-1}, \cdots, b_{n-1} / \kappa_{n-1}\right)$, it follows that $a=\sum_{r=0}^{s_{n}-1} a(r)$.

By induction, $\nu_{d^{\prime}}-1-c_{r} \in R_{d^{\prime}-a(r)}\left(b_{1} / \kappa_{n-1}, \cdots, b_{n-1} / \kappa_{n-1}\right), r=0,1, \cdots$, $s_{n}-1$. We have $\nu_{d}-1=\kappa_{n-1}\left(\nu_{d^{\prime}}-1\right)+\left(s_{n} \kappa_{n-1}-1\right) b_{n}$. Therefore, if $\nu_{d^{\prime}}-1-c_{r}$ $=\sum_{i=1}^{n-1} z_{i}\left(b_{i} / \kappa_{n-1}\right)$, then $\nu_{d}-1-c=\sum_{i=1}^{n-1} z_{i} b_{i}+\left(s_{n} \kappa_{n-1}-1-\left(z_{n}+r \kappa_{n-1}\right)\right) b_{n}$. This shows that every proper representation of $\nu_{d^{\prime}}-1-c_{r}$ gives rise to a representation of $c$ as a proper combination of $b_{1}, b_{2}, \cdots, b_{n}$, and hence there are at least $\sum_{r=0}^{s_{n}-1}\left(d^{\prime}-a(r)\right)=d-a$ proper representations of $\nu_{d}-1-c$.

On the other hand, if $\nu_{d}-1-c=\sum_{i=1}^{n} y_{i} b_{i}$, then $y_{n}=s_{n} \kappa_{n-1}-1-\left(z_{n}+r \kappa_{n-1}\right)$ for some $r=0,1, \cdots, s_{n}-1$, and so $\nu_{d^{\prime}}-1-c_{r}=\sum_{i=1}^{n-1} y_{i}\left(b_{i} / \kappa_{n-1}\right)$. Thus every representation of $\nu_{d}-1-c$ arises from one of $\nu_{d^{\prime}}-1-c_{r}$ for some integer $r=0,1, \cdots, s_{n}-1$. Hence $\nu_{d}-1-c \in R_{d-a}\left(b_{1}, b_{2}, \cdots, b_{n}\right)$.

(j). Proof of (c).

It follows from (i) that for every integer $c<\nu_{d}, \chi(c)+\chi\left(\nu_{d}-1-c\right)=d$. We consider the pairs of integers: $c, \nu_{d}-1-c$. If $\nu_{d}$ is an even integer, we have exactly $2^{-1} \nu_{d}$ distinct pairs, and hence $\sum_{c=0}^{\nu_{d}-1} \chi(c)=d\left(2^{-1} \nu_{d}\right)$. If $\nu_{d}$ is odd, there are $2^{-1}\left(\nu_{d}-1\right)$ distinct pairs in addition to the single integer $2^{-1}\left(\nu_{d}-1\right)$ which is paired with itself. Since (i) implies that $2^{-1}\left(\nu_{d}-1\right) \in R_{d / 2}\left(b_{1}, b_{2}, \cdots, b_{n}\right)$, we conclude that $\sum_{c=0}^{\nu_{d}-1} \chi(c)=d\left(2^{-1}\left(\nu_{d}-1\right)\right)+2^{-1} d=2^{-1} d \nu_{d}$. This completes the proof of the theorem.

We conclude with two arithmetic results which we shall need in the next section.

LEMma 1. Assume $b_{i}>s_{i-1} b_{i-1}\left(\kappa_{i-2} / \kappa_{i-1}\right), i=3,4, \cdots, n$. Then there exist non-negative integers $\gamma_{i j}, j=1,2, \cdots, n-1 ; i=j+1, \cdots, n$, such that

(a) $\left(\kappa_{i-1} / \kappa_{i}\right) b_{i}=\sum_{j=1}^{i-1} \gamma_{i j} b_{j}, i=2,3, \cdots, n$;

(b) $0 \leqq \gamma_{i j}-\sum_{m=j+1}^{i-1} \gamma_{m j}\left(s_{m}-1\right)<\left(\kappa_{j-1} / \kappa_{j}\right), \quad j=2, \cdots, n-1 ; i=j+1$, $\cdots, n$;

(c) $0 \leqq \gamma_{i 1}-\sum_{m=2}^{i-1} \gamma_{m 1}\left(s_{m}-1\right), i=2,3, \cdots, n$.

Proof. We first prove the assertion:

(d) Let $c$ be an arbitrary integer divisible by $\kappa_{n}$ and let $a_{2}, a_{3}, \cdots, a_{n}$ be given integers. Then we can write $c=\sum_{i=1}^{n} z_{i} b_{i}$ with $a_{i} \leqq z_{i}<a_{i}+\left(\kappa_{i-1} / \kappa_{i}\right)$, $i=2,3, \cdots, n$.

Since $\left(\kappa_{n-1} / \kappa_{n}, b_{n} / \kappa_{n}\right)=1,\left(c / \kappa_{n}\right)-z_{n}\left(b_{n} / \kappa_{n}\right) \equiv 0\left(\bmod \kappa_{n-1} / \kappa_{n}\right)$ for some $z_{n}$ such that $a_{n} \leqq z_{n}<a_{n}+\left(\kappa_{n-1} / \kappa_{n}\right)$. Hence $c-z_{n} b_{n} \equiv 0\left(\bmod \kappa_{n-1}\right)$, and (d) follows by induction.

As for the lemma itself, if $n=2$ we have $\left(b_{1} / \kappa_{2}\right) b_{2}=\left(b_{2} / \kappa_{2}\right) b_{1}$. Thus $\gamma_{21}=\left(b_{2} / \kappa_{2}\right)$. We proceed by induction. Applied to the integers $b_{1}, b_{2}, \cdots, b_{n-1}$, we may assume the existence of integers $\gamma_{i j}, j=1,2, \cdots, n-2 ; i=j$ $+1, \cdots, n-1$ which satisfy (a), (b), and (c).

Let $a_{j}=\sum_{m=j+1}^{n-1} \gamma_{m j}\left(s_{m}-1\right), j=1,2, \cdots, n-2$, and let $a_{n-1}=0$. Since $\left(\kappa_{n-1} / \kappa_{n}\right) b_{n}$ is divisible by $\kappa_{n-1}$, we can apply (d) to it and to the integers $a_{2}, a_{3}, \cdots, a_{n-1}$, as just defined. We conclude that there exist integers 
$\gamma_{n 1}, \gamma_{n 2}, \cdots, \gamma_{n n-1}$ such that $\left(\kappa_{n-1} / \kappa_{n}\right) b_{n}=\sum_{j=1}^{n-1} \gamma_{n j} b_{j}$ and $0 \leqq \gamma_{n j}-a_{j}$ $<\left(\kappa_{j-1} / \kappa_{j}\right), j=2,3, \cdots, n-1$. Hence the integers $\gamma_{n j}, j=1,2, \cdots, n-1$, satisfy (a) and (b). The proof will be completed by showing that $\boldsymbol{\gamma}_{n 1}$ satisfies (c) 一that is, $\gamma_{n i} \geqq a_{1}$.

$$
\gamma_{n 1} b_{1}=\left(\kappa_{n-1} / \kappa_{n}\right) b_{n}-\sum_{j=2}^{n-1} \gamma_{n j} b_{j} \geqq\left(\kappa_{n-1} / \kappa_{n}\right) b_{n}-\sum_{j=2}^{n-1}\left(a_{j}+\left(\kappa_{j-1} / \kappa_{j}\right)-1\right) b_{j},
$$

whence it will suffice to prove that $\left(\kappa_{n-1} / \kappa_{n}\right) b_{n}-\sum_{j=2}^{n-1}\left(a_{j}+\left(\kappa_{j-1} / \kappa_{j}\right)-1\right) b_{j}$ $\geqq a_{1} b_{1}$. Using the definition of the integers $a_{j}$ and condition (a), this last inequality can be rewritten, as follows:

(e) $b_{n}-\sum_{i=2}^{n-1}\left(s_{i}\left(\kappa_{i-1} / \kappa_{i}\right)-1\right) b_{i} \geqq 0$.

Since by hypothesis $b_{i}>s_{i-1} b_{i-1}\left(\kappa_{i-2} / \kappa_{i-1}\right), i=3, \cdots, n$, it follows that

$$
b_{n}>s_{n-1} b_{n-1}\left(\kappa_{n-2} / \kappa_{n-1}\right)=\left[s_{n-1}\left(\kappa_{n-2} / \kappa_{n-1}\right)-1\right] b_{n-1}+b_{n-1},
$$

and (e) follows by induction. Q.E.D.

Let $R\left(b_{1}, \cdots, b_{n}\right)$ denote the set of integers which may be written as a linear combination of $b_{1}, \cdots, b_{n}$ with arbitrary non-negative integers.

Lemma 2. Assume $b_{i}>s_{i-1} b_{i-1}\left(\kappa_{i-2} / \kappa_{i-1}\right), i=3, \cdots, n$. Then

(a) If $c \in R\left(b_{1}, b_{2}, \cdots, b_{n}\right)$, then $c \in R_{a}\left(b_{1}, b_{2}, \cdots, b_{n}\right)$ with $a \geqq 1$.

(b) If $c>\left(b_{n} / \kappa_{n}\right)\left(\kappa_{n-1} / \kappa_{n}\right), \kappa_{n} c \in R\left(b_{1}, \cdots, b_{n}\right)$.

(c) Define $t_{i}=t_{i-1} s_{i-1}\left(\kappa_{i-2} / \kappa_{i-1}\right), i=3, \cdots, n$, with $t_{2}$ an arbitrary positive integer. Then $\left(\kappa_{n-1} / \kappa_{n}\right) t_{n} \geqq t_{2}+\sum_{i=2}^{n-1}\left(s_{i}\left(\kappa_{i-1} / \kappa_{i}\right)-1\right) t_{i}$.

Proof. If $c \in R\left(b_{1}, \cdots, b_{n}\right), c=\sum_{i=1}^{n} y_{i} b_{i}, y_{i} \geqq 0$. Write $y_{n}=r_{n}\left(\kappa_{n-1} / \kappa_{n}\right)$ $+z_{n}, 0 \leqq z_{n}<\left(\kappa_{n-1} / \kappa_{n}\right), r_{n} \geqq 0$. Since $\left(\kappa_{n-1} / \kappa_{n}\right) b_{n}=\sum_{j=1}^{n-1} \gamma_{n j} b_{j}$ and $\gamma_{n j} \geqq 0$, $j=1,2, \cdots, n$, it follows that $c=\sum_{i=1}^{n-1} y_{i}^{\prime} b_{i}+z_{n} b_{n}$ with $y_{i}^{\prime} \geqq 0, i=1,2, \cdots$, $n-1$. Thus by induction we conclude that that $c=\sum_{i=1}^{n} z_{i} b_{i}, z_{i} \geqq 0, i=1,2$, $\cdots, n$, and $z_{i}<\left(\kappa_{i-1} / \kappa_{i}\right), i=2, \cdots, n$. Thus $c$ may be written as a proper combination of $b_{1}, b_{2}, \cdots, b_{n}$ in at least one way, and hence $c$ $\in R_{a}\left(b_{1}, b_{2}, \cdots, b_{n}\right)$ with $a \geqq 1$.

Take $n=2$, and suppose $c>\left(b_{2} / \kappa_{2}\right)\left(b_{1} / \kappa_{2}\right)$. As in Theorem 4, (d), $c=y_{1}\left(b_{1} / \kappa_{2}\right)+y_{2}\left(b_{2} / \kappa_{2}\right), y_{i} \geqq 0, i=1,2$; whence $\kappa_{2} c \in R\left(b_{1}, b_{2}\right)$. We use induction. If $c>\left(b_{n} / \kappa_{n}\right)\left(\kappa_{n-1} / \kappa_{n}\right)$, there exists an integer $z_{n}$ with $0 \leqq z_{n}<\left(\kappa_{n-1} / \kappa_{n}\right)$ such that $c-z_{n}\left(b_{n} / \kappa_{n}\right) \equiv 0\left(\bmod \left(\kappa_{n-1} / \kappa_{n}\right)\right)$. Denote the integer $\left(\kappa_{n} c-z_{n} b_{n}\right) / \kappa_{n-1}$ by $c^{\prime}$. Since $c>\left(b_{n} / \kappa_{n}\right)\left(\kappa_{n-1} / \kappa_{n}\right)$ and $z_{n} \leqq\left(\kappa_{n-1} / \kappa_{n}\right)-1$, it follows that $c^{\prime}$ $>\left[\left(b_{n} \kappa_{n-1} / \kappa_{n}\right)-\left(\left(\kappa_{n-1} / \kappa_{n}\right)-1\right) b_{n}\right] / \kappa_{n-1}=\left(b_{n} / \kappa_{n}\right) . \quad b_{n}>s_{n-1} b_{n-1}\left(\kappa_{n-2} / \kappa_{n-1}\right)$ by hypothesis, and $s_{n-1} \geqq 1$, so that $c^{\prime}>\left(b_{n-1} / \kappa_{n-1}\right)\left(\kappa_{n-2} / \kappa_{n-1}\right)$. By induction, $\kappa_{n-1} c^{\prime} \in R\left(b_{1}, \cdots, b_{n-1}\right)$. Since $\kappa_{n-1} c^{\prime}=\kappa_{n} c-z_{n} b_{n}$, (b) is proved.

(c) is established in the same way as part (e) of Lemma 1.

4. A generalization of a theorem of Seidenberg. Seidenberg [7] has given a detailed analysis of the valuation ideals in a polynomial ring in two independent variables over an algebraically closed ground field. As a special 
case of his theory one can obtain the corresponding analysis for the valuation ideals in a nonhomogeneous coordinate ring of a plane algebraic curve (over an algebraically closed ground field). The main tool in his work is the construction, for a given valuation, of a special set of polynomials intrinsically associated with the valuation (Theorem 6).

We shall generalize this construction for valuations of an algebraic function field of one variable over an arbitrary ground field and for discrete valuations of an algebraic number field.

Denote by $\pi_{1}$ the irreducible monic polynomial in $X$ with coefficients in $k$ or the rational prime integer which generates the prime ideal $\mathfrak{p}_{0}$ in $\mathfrak{D}_{0}$. Let $\pi_{1}, \pi_{2}$ be a basis for the prime ideal $\mathfrak{p}$ in $\mathfrak{o}$. If we assume that $\pi_{2}$ is a monic polynomial in $y$ whose coefficients are, in the one case, in $k[X]$ of degree less than $\operatorname{deg}_{X} \pi_{1}$ or, in the other case, non-negative integers less than $\pi_{1}$, then $\pi_{2}$ is uniquely determined. Clearly every element of $\mathfrak{D}$ is congruent modulo $\mathfrak{p}$ to a polynomial in $y$ of degree less than $\operatorname{deg}_{v} \pi_{2}$. Let $\eta_{i}, i=1,2, \cdots, d_{\mathfrak{p}}$, be elements of $\mathrm{D}$ which form a basis for $\mathrm{D}$ modulo $\mathfrak{p}$ and such that $\operatorname{deg}_{y} \eta_{i}<\operatorname{deg}_{\nu} \pi_{2}$.

THEOREM 5. Let $\boldsymbol{v}$ be a valuation of $K$ with center $\mathfrak{p}$ in $\mathbf{0}$. It is possible to construct a set of elements $\pi_{3}, \pi_{4}, \cdots, \pi_{n}$ in 0 which together with $\pi_{1}, \pi_{2}$ satisfy all the following conditions:

(a) $\pi_{i}$ may be written as a monic polynomial in $y$ with coefficients in $\mathrm{D}_{0}$, $i=3, \cdots, n$. If $\operatorname{deg}_{y} \pi_{i}=t_{i}, i=2, \cdots, n$, and if $v\left(\pi_{i}\right)=b_{i}, i=1,2, \cdots, n$, while $\kappa_{i}=\left(b_{1}, \cdots, b_{i}\right)$, there exist positive integers $s_{2}, s_{3}, \cdots, s_{n}$ such that

(b) $t_{2}=s_{1}$ and $t_{i}=t_{i-1} s_{i-1}\left(\kappa_{i-2} / \kappa_{i-1}\right), i=3, \cdots, n$;

(c) $b_{i}>s_{i-1} b_{i-1}\left(\kappa_{i-2} / \kappa_{i-1}\right), i=3, \cdots, n$;

(d) If $d=$ the degree of $v$, then $d=d_{\mathfrak{p}} \prod_{i=2}^{n} s_{i}$; moreover,

(e) $\left(b_{1}, b_{2}, \cdots, b_{n}\right)=1$;

(f) If $\phi \in 0, v(\phi) \in R\left(b_{1}, b_{2}, \cdots, b_{n}\right)$;

(g) If $\left\{\mathfrak{q}_{j}\right\}$ denotes the sequence of $v$-ideals in $\mathrm{o}$, then the monomials $\eta_{h} \prod_{i=1}^{n} \pi_{i}^{a_{i}}$ with $h=1,2, \cdots, d_{\mathfrak{p}}$ and $a_{i}<s_{i}\left(\kappa_{i-1} / \kappa_{i}\right), i=2,3, \cdots, n$, of value $v\left(\mathfrak{q}_{j}\right)$ form an independent basis for $\mathfrak{q}_{j}$ modulo $\mathfrak{q}_{j+1}$.

Proof. For brevity, we call a monomial $\eta_{h} \prod_{i=1}^{r} \pi_{i}^{a_{i}}$ with $a_{i}<s_{i}\left(\kappa_{i-1} / \kappa_{i}\right)$, $i=2,3, \cdots, r$, a canonical product of $\pi_{1}, \cdots, \pi_{r}$. If $\phi \in \mathcal{D}$ can be expressed as a linear combination (with coefficients in $k$ or in $I$, as the case may be) of canonical products $\pi_{1}, \cdots, \pi_{r}$, we shall write $\phi \in R\left(\pi_{1}, \cdots, \pi_{r}\right)$.

It will be convenient to add the statement:

(h) if $\operatorname{deg}_{y} \phi<t_{i}$ for some $i$, then $\phi \in R\left(\pi_{1}, \cdots, \pi_{i-1}\right)$ and $v(\phi)$ $\in R\left(b_{1}, \cdots, b_{i-1}\right)$.

If $\operatorname{deg}_{y} \phi<t_{2}$, it is clear that $\phi=\sum_{i=1}^{m} c_{i} \eta_{h(i)} \pi_{1}^{a_{i}}, a_{i} \geqq 0$, and $c_{i} \in k$ or $c_{i} \in I$, $i=1,2, \cdots, m$, whence (h) holds in this case. Assume that $\pi_{3}, \cdots, \pi_{r}$ have been constructed satisfying (a), (b), (c), and (h). Then $b_{1}, \cdots, b_{r}$ satisfy the hypothesis of Lemmas 1 and 2 . Let $\sigma_{i}=\prod_{j=1}^{i-1} \pi_{j}^{\left(\gamma_{i j}\right)}, i=2,3, \cdots, r$, where the $\gamma_{i j}$ are the integers whose existence is asserted in Lemma 1. Also let 
$\psi_{i}=\left(\pi_{i}^{\left(\kappa_{i-1} / \kappa_{i}\right)} / \sigma_{i}\right), i=2,3, \cdots, r$. By the definition of $\gamma_{i j}, v\left(\sigma_{i}\right)=\left(\kappa_{i-1} / \kappa_{i}\right) b_{i}$ and $\boldsymbol{v}\left(\psi_{i}\right)=0, i=2,3, \cdots, r$.

As part of our induction hypothesis, we shall assume: if $\alpha_{2}=\bar{y}$ and $\alpha_{i+1}$ $=\bar{\psi}_{i}, i=2,3, \cdots, r(-$ denotes the residue, modulo the ideal of nonunits, of an element of the valuation ring of $\boldsymbol{v})$, then

(i) $s_{i}=\left[k_{0}\left(\alpha_{2}, \cdots, \alpha_{i+1}\right): k_{0}\left(\alpha_{2}, \cdots, \alpha_{i}\right)\right], i=2,3, \cdots, r-1$.

We define $s_{r}$ by (i) and proceed to construct an element $\pi_{r+1}$ in $\mathbf{D}$ satisfying (a), (b), (c) and (h). There exists a unique polynomial $g_{r}\left(\alpha_{2}, \cdots, \alpha_{r}, Z\right)$ with coefficients in $k_{0}$, monic in $Z$ of degree $s_{r}$, whose degree in $\alpha_{i}<s_{i-1}$, $i=2,3, \cdots, r$, and which is zero for $Z=\alpha_{r+1}$. Let $g_{r}^{\prime}\left(y, \psi_{2}, \cdots, \psi_{r-1}, Z\right)$ be a monic polynomial obtained from $g_{r}$ by replacing $\alpha_{2}$ by $y, \alpha_{i+1}$ by $\psi_{i}, i$ $=2, \cdots, r-1$, and each coefficient by a representative in $\mathfrak{D}_{0}$.

It follows directly from part (b) of Lemma 1 that $\prod_{i=1}^{r-1} \sigma_{i}^{s_{i}-1}$ divides $\sigma_{r}$ in $\mathfrak{D}$; and hence by the construction of $g_{r}^{\prime}$ and the definition of $\psi_{i}$, $\sigma_{r}^{s_{r}} \cdot g_{r}^{\prime}\left(y, \psi_{2}, \cdots, \psi_{r}\right) \in \mathbf{0}$.

We define $\pi_{r+1}$ to be $\sigma_{r}^{s_{r}} \cdot g_{r}^{\prime}\left(y, \psi_{2}, \cdots, \psi_{r}\right)$. By construction

$$
v\left(g_{r}^{\prime}\left(y, \psi_{2}, \cdots, \psi_{r}\right)\right)>0 \text {, }
$$

whence $\boldsymbol{v}\left(\pi_{r+1}\right)>\boldsymbol{v}\left(\sigma_{r}^{s_{r}}\right)=s_{r}\left(\kappa_{r-1} / \kappa_{r}\right) b_{r}$. Hence (c) holds for $\pi_{r+1}$.

The proof of (a) and (b) depends upon the following assertion:

(j) $\operatorname{deg}_{\nu} \sigma_{i}<\operatorname{deg}_{\nu} \pi_{i}^{\left(\kappa_{i-1} / \kappa_{i}\right)}=\left(\kappa_{i-1} / \kappa_{i}\right) t_{i}, i=2,3, \cdots, r$. For $i=2, \sigma_{2}$ is a power of $\pi_{1}$ whence $\operatorname{deg}_{y} \sigma_{2}=0$. Assume $(\mathrm{j})$ for $\sigma_{2}, \cdots, \sigma_{r-1}$. By definition, $\operatorname{deg}_{y} \sigma_{i}=\sum_{j=2}^{i-1} \gamma_{i j} t_{j}, i=3, \cdots, r$. Combining these relations with the inequalities $\gamma_{r j} \leqq\left(\kappa_{j-1} / \kappa_{j}\right)-1+\sum_{e=1+1}^{r-1} \gamma_{e_{j}}\left(s_{e}-1\right), j=2,3, \cdots, r-1$, a direct computation yields $\left.\operatorname{deg}_{y} \sigma_{r}<\sum_{i=2}^{r-1}\left[\left(s_{i}-1\right) \operatorname{deg}_{y} \sigma_{i}+\left(\kappa_{i-1} / \kappa_{i}\right)-1\right) t_{i}\right]$. Applying (j) for $i<r$, we obtain $\operatorname{deg}_{y} \sigma_{r}<\sum_{i=2}^{r-1}\left(s_{i}\left(\kappa_{i-1} / \kappa_{i}\right)-1\right) t_{i}<\left(\kappa_{r-1} / \kappa_{r}\right) t_{r}$ by Lemma 2.

The conclusion to be drawn from ( $j$ ) is the following: the term of highest degree in $y$ in $\pi_{r+1}$ is either less than or equal to the degree in $y$ of $\theta_{1}=y^{s_{1}-1} \sigma_{r}^{s_{r}} \prod_{i=2}^{r} \psi_{i}^{s_{i}-1}$, or is equal to the degree in $y$ of $\theta_{2}=\pi_{r}^{s_{r}\left(\kappa_{r-1} / \kappa_{r}\right)}$, since $\operatorname{deg}_{y} \theta_{1}=\operatorname{deg}_{y} \sigma_{r}-\sum_{i=2}^{r-1}\left[\left(s_{i}-1\right) \operatorname{deg}_{y} \sigma_{i}\right]+s_{1}-1+\sum_{i=2}^{r-1}\left(s_{i}-1\right)\left(\kappa_{i-1} / \kappa_{i}\right) t_{i}$. It follows from Lemma 1 that

$$
\operatorname{deg}_{y} \sigma_{r}-\sum_{i=2}^{r-1}\left(s_{i}-1\right) \operatorname{deg}_{y} \sigma_{i} \leqq \sum_{i=2}^{r-1}\left(\left(\kappa_{i-1} / \kappa_{i}\right)-1\right) t_{i}
$$

whence $\operatorname{deg}_{y} \theta_{1} \leqq s_{1}-1+\sum_{i=2}^{r-1}\left(s_{i}\left(\kappa_{i-1} / \kappa_{i}\right)-1\right) t_{i}$. Since $s_{1}=t_{2}$ and $s_{r} \geqq 1$, it follows from Lemma 2 that $\operatorname{deg}_{y} \theta_{1}<s_{r}\left(\kappa_{r-1} / \kappa_{r}\right) t_{r}=\operatorname{deg}_{y} \theta_{2}$.

Thus $\pi_{r+1}$ is indeed monic in $y$ of degree $t_{r+1}=s_{r}\left(\kappa_{r-1} / \kappa_{r}\right) t_{r}$.

Suppose $\phi \in 0$ and $\operatorname{deg}_{y} \phi<t_{r+1}$. Since $\pi_{r}$ is monic in $y$ we may write $\phi=\phi_{0}+\phi_{1} \pi_{r} \cdots \phi_{e} \pi_{r}^{e}, e<s_{r}\left(\kappa_{r-1} / \kappa_{r}\right)$, and $\operatorname{deg}_{\nu} \phi_{i}<t_{r}$ (by (b)). Hence by induction $\phi_{i} \in R\left(\pi_{1}, \cdots, \pi_{r-1}\right)$ and $\phi \in R\left(\pi_{1}, \cdots, \pi_{r}\right)$. The proof that $v(\phi)$ $\in R\left(b_{1}, \cdots, b_{r}\right)$ depends upon a portion of $(\mathrm{g})$, namely: 
(k) The canonical products of $\pi_{1}, \cdots, \pi_{r}$ of value $v\left(\mathfrak{q}_{j}\right)$ are linearly independent modulo $\mathfrak{q}_{j+1}$.

For we already know that $\phi$ is a linear combination of canonical products of $\pi_{1}, \cdots, \pi_{r}$, and $(\mathrm{k})$ shows that $v(\phi)$ is equal to the minimum value of the canonical products which occur in the expression for $\phi$. Hence $v(\phi)$ $\in R\left(b_{1}, \cdots, b_{r}\right)$.

To prove $(\mathrm{k})$, proceed inductively, observing that $(\mathrm{k})$ certainly holds for the canonical products of $\pi_{1}$ (if any) of value $v\left(\mathfrak{q}_{j}\right)$. Assume (k) for canonical products of $\pi_{1}, \cdots, \pi_{r-1}$, and let $\zeta=\sum_{i=1}^{m} c_{i} \zeta_{i}$ be a linear combination of canonical products $\zeta_{i}$ of $\pi_{1}, \cdots, \pi_{r}$ in which $v\left(\mathfrak{q}_{j}\right)=v\left(\zeta_{i}\right), i=1,2, \cdots, m$. We must prove that $\zeta \notin \mathfrak{q}_{j+1}$.

We first prove:

(l) A power product $\chi=\prod_{i=1}^{r} \pi_{i}^{a_{i}}$ with $a_{i}>0,=0$, or $<0$, and with $v(\chi)$ $=0$ may be expressed in the form $\prod_{i=2}^{r} \psi_{i}^{m_{i}}, m_{i}>0,=0$, or $<0$. In fact, $\sum_{i=1}^{r} a_{i} b_{i}=0$, whence $a_{r}=m_{r}\left(\kappa_{r-1} / \kappa_{r}\right)$ for some integer $m_{r}>0,=0$, or $<0$. Thus $\chi=\psi_{r}^{m_{r}} \sigma_{r}^{m_{r}} \prod_{i-1}^{r-1} \pi_{i}^{a_{i}}$. Since $\sigma_{r}^{m_{r}} \prod_{i=1}^{r-1} \pi_{i}^{a_{i}}$ is a power product of $\pi_{1}, \cdots, \pi_{r-1}$ and since $v\left(\psi_{r}\right)=0$, (l) follows readily by induction. An immediate corollary of (l) is that $\overline{\left(\zeta_{i} / \zeta_{k}\right)} \in k_{0}\left(\alpha_{2}, \cdots, \alpha_{r+1}\right)$ for all $i, k=1,2, \cdots, m$.

If $e_{i}=\exp \pi_{r}$ in $\zeta_{i}$, we may assume that $\max \left(e_{1}, \cdots, e_{m}\right)>0$ and, for definiteness, that $e_{1}=\min \left(e_{1}, \cdots, e_{m}\right)$. It follows at once from the preceding remark that $\overline{\left(\zeta / \zeta_{1}\right)}$ is a polynomial $P\left(\alpha_{r+1}\right)$ in $\alpha_{r+1}$ with coefficients in $k_{0}\left(\alpha_{2}, \cdots, \alpha_{r}\right)$. Since $e_{i}<s_{r}\left(\kappa_{r-1} / \kappa_{r}\right)$ for each $i, \operatorname{deg}_{z} P(Z)<s_{r}$. But if $\zeta$ $\in \mathfrak{q}_{j+1}, \boldsymbol{v}(\zeta)>\boldsymbol{v}\left(\zeta_{1}\right)$ whence $\overline{\left(\zeta / \zeta_{1}\right)}=0=P\left(\alpha_{r+1}\right)$, in contradiction to the fact that $\alpha_{r+1}$ satisfies no polynomial of degree $<s_{r}$ with coefficients in $k_{0}\left(\alpha_{2}, \cdots, \alpha_{r}\right)$.

We have thus succeeded in constructing an element $\pi_{r+1}$ which satisfies (a), (b), (c), and (h). The remainder of the proof consists in showing that after a finite number of such constructions (d), (e), (f), (g) can also be made to hold.

It may happen first of all, at some step in this process that $\pi_{r+m+1}=0$. In this case, we assert $\pi_{1}, \cdots, \pi_{r+m}$ satisfy all the conditions of the theorem. Indeed every $\phi \in \mathfrak{o}$ can then be expressed as a polynomial in $y$ with coefficients in $D_{0}$ of degree $<t_{r+m+1}\left({ }^{3}\right)$. By $(\mathrm{h}), \boldsymbol{v}(\phi) \in R\left(b_{1}, \cdots, b_{r+m}\right)$ and $\phi$ $\in R\left(\pi_{1}, \cdots, \pi_{r+m}\right)$ which proves (f) and, in view of (k), also proves $(\mathrm{g})$. Moreover, if $\phi \in K, v(\phi)=\sum_{i=1}^{n} a_{i} b_{i}, a_{i}>0,=0$, or $<0$, and since $K$ contains an element of value 1 , it follows that $\left(b_{1}, b_{2}, \cdots, b_{r+m}\right)=1$. Finally (e) implies that $k_{0}\left(\alpha_{2}, \cdots, \alpha_{r+m+1}\right)$ is the entire residue field of $v$, whence $d=d_{\mathfrak{p}} \prod_{i=2}^{r+m} s_{i}$, proving (d).

Suppose now that $\kappa_{r}=1$. If $\phi \in \mathfrak{0}$, and $\boldsymbol{v}(\phi)>s_{r}\left(\kappa_{r-1} / \kappa_{r}\right) b_{r}$, it follows from Lemma 2 that $\boldsymbol{v}(\phi) \in R\left(b_{1}, \cdots, b_{r}\right)$. On the other hand, if $\phi \in \mathfrak{0}$, and $\boldsymbol{v}(\phi)$ $\leqq s_{r}\left(\kappa_{r-1} / \kappa_{r}\right) b_{r}, \phi=\phi_{1}+\phi_{2} \pi_{r+1}, \phi_{i} \in \mathfrak{D}, i=1,2$, and $\operatorname{deg}_{y} \phi_{1}<t_{r+1}$. Since $v\left(\pi_{r+1}\right)$ $>s_{r} \kappa_{r-1} b_{r}, v(\phi)=v\left(\phi_{1}\right) \in R\left(b_{1}, \cdots, b_{r}\right)$ by (h). Therefore (e) implies (f).

(3) $t_{r+m+1}$ denotes the formal degree of $\pi_{r+m+1}$ as a polynomial in $y$. 
To prove (e) suppose $\kappa_{r}>1$. Then there exists $\phi \in_{0}$ such that $v(\phi)$ $\notin R\left(b_{1}, \cdots, b_{r}\right)$. Suppose $\pi_{r+i}, i=1,2, \cdots, e$, have been constructed and that $b_{r+i}=v\left(\pi_{r+i}\right) \in R\left(b_{1}, \cdots, b_{r}\right), i=1,2, \cdots, e$. Assume $b_{r+i}>v(\phi)$ for some $i$. As above, $\phi=\phi_{1}+\phi_{2} \pi_{r+i}, \operatorname{deg}_{y} \phi_{1}<t_{r+i}$. Hence $v(\phi)=v\left(\phi_{1}\right)$ $\in R\left(b_{1}, \cdots, b_{r+i-1}\right)=R\left(b_{1}, \cdots, b_{r}\right)$, contrary to the choice of $\phi$. Thus if $b_{r+i} \in R\left(b_{1}, \cdots, b_{r}\right), b_{r+i}<v(\phi)$. Since $b_{r+i+1}>b_{r+i}$, there exists an integer $m$ such that $b_{r+m}=v\left(\pi_{r+m}\right) \notin R\left(b_{1}, \cdots, b_{r}\right)$. We shall show that $\kappa_{r+m}<\kappa_{r}$. Since certainly $b_{r+m}>b_{r}\left(\kappa_{r-1} / \kappa_{r}\right),\left(b_{r+m} / \kappa_{r+m}\right)>\left(b_{r} / \kappa_{r}\right)\left(\kappa_{r-1} / \kappa_{r+m}\right)$. If $\kappa_{r+m}=\kappa_{r}$, we conclude immediately from Lemma 2 that $b_{r+m} \in R\left(b_{1}, \cdots, b_{r}\right)$, a contradiction; whence $\kappa_{r+m}<\kappa_{r}$. Consequently a finite number of constructions of elements $\pi_{i}, i=1,2, \cdots, n$, will be sufficient to insure that $\left(b_{1}, b_{2}, \cdots, b_{n}\right)$ $=1$.

Assume that the residue field of $\boldsymbol{v}$ contains an element $\alpha^{\prime}$ not in $k_{0}\left(\alpha_{2}, \cdots, \alpha_{r+1}\right)$. If $\phi_{1} / \phi_{2}$ is a representative of $\alpha^{\prime}$ in $K, \phi_{i} \in 0, i=1,2$, let $b^{\prime}=v\left(\phi_{1}\right)=v\left(\phi_{2}\right)$. Construct $\pi_{r+i}, i=1,2, \cdots, m+1$, so that $b^{\prime}<b_{r+m+1}$. As above, write $\phi_{i}=\phi_{i}^{\prime}+\phi_{i}^{\prime \prime} \pi_{r+m+1}, \operatorname{deg}_{y} \phi_{i}^{\prime}<t_{r+m+1}, i=1,2$. Then $b^{\prime}=v\left(\phi_{i}^{\prime}\right)$, $i=1,2$, and by (1) the residue of $\phi_{1}^{\prime} / \phi_{2}^{\prime}$ is in $k_{0}\left(\alpha_{2}, \cdots, \alpha_{r+m+1}\right)$. But clearly this residue is $\alpha^{\prime}$, whence $\alpha^{\prime} \in k_{0}\left(\alpha_{2}, \cdots, \alpha_{r+m+1}\right)$. We conclude from the definition of $s_{i}$ and the preceding results that we can construct elements $\pi_{1}, \pi_{2}, \cdots, \pi_{n}$ in $\mathrm{o}$ which satisfy (a)-(f).

To complete the proof we need only verify that the canonical products of $\pi_{1}, \pi_{2}, \cdots, \pi_{n}$ of value $v\left(\mathfrak{q}_{j}\right)$ span $\mathfrak{q}_{j}$ modulo $\mathfrak{q}_{j+1}$. This will establish $(\mathrm{g})$ since the independence of these canonical products has already been proved. If $v\left(\mathfrak{q}_{j}\right) \leqq s_{n} \kappa_{n-1} b_{n}$ and if $\phi \in \mathfrak{q}_{j}$, write $\phi=\phi_{1}+\phi_{2} \pi_{n}$, with $\operatorname{deg}_{y} \phi_{1}<t_{n}$. Then $\phi \equiv \phi_{1}\left(\bmod \mathfrak{q}_{j+1}\right)$ and, by $(\mathrm{h}), \phi_{1} \in R\left(\pi_{1}, \pi_{2}, \cdots, \pi_{n-1}\right)$.

Suppose that $v\left(\mathfrak{q}_{j}\right)>s_{n} \kappa_{n-1} b_{n}$. Using equation (e) of Lemma 1, we have

$$
s_{n} \kappa_{n-1} b_{n}>\left[\sum_{i=2}^{n}\left(s_{i} \kappa_{i-1} / \kappa_{i}-1\right) b_{i}\right]-\left(b_{1}-1\right)=\nu_{d^{\prime}}\left(b_{1}, b_{2}, \cdots, b_{n}\right),
$$

where $d^{\prime}=\prod_{i=2}^{n} s_{i}$. It follows then from Theorem 4 that $v\left(\mathfrak{q}_{j}\right)$ $\in R_{d^{\prime}}\left(b_{1}, b_{2}, \cdots, b_{n}\right)$. Let $v\left(\mathfrak{q}_{j}\right)=\sum_{i=1}^{n} a_{i m} b_{i}, m=1,2, \cdots, d^{\prime}$, be the distinct representations of $v\left(\mathfrak{q}_{j}\right)$ as a proper combination of $b_{1}, b_{2}, \cdots, b_{n}$. Then the $d$ monomials $\eta_{h} \prod_{i=1}^{n} \pi_{i}^{\left(a_{i m}\right)}, h=1,2, \cdots, d_{\mathfrak{p}} ; m=1,2, \cdots, d^{\prime}$, are distinct canonical products of value $\boldsymbol{v}\left(\mathfrak{q}_{j}\right)$, which are linearly independent modulo $\mathfrak{q}_{j+1}$ by $(\mathrm{k})$. We conclude that $\operatorname{dim} \mathfrak{q}_{j} / \mathfrak{q}_{j+1}=d$ and that these $d$ canonical products span $\mathfrak{q}_{j}$ modulo $\mathfrak{q}_{j+1}$. Q.E.D.

\section{The main theorem.}

ThEOREM 6. $d\left(\mathfrak{G}_{\mathfrak{p}}\right)=2 \delta\left(\mathfrak{G}_{\mathfrak{p}}\right)$, or equivalently $\operatorname{dim} \overline{\mathfrak{D}}_{\mathfrak{p}} / \mathfrak{G}_{\mathfrak{p}}=2 \operatorname{dim} \mathfrak{D}_{\mathfrak{p}} / \mathbb{C}_{\mathfrak{p}}$.

Proof. Because of the one-one correspondence between complete $\mathfrak{p}$-ideals

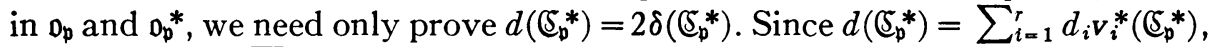
and since $\mathfrak{C}_{p}^{*}=\sum_{i=1}^{r} \mathfrak{S}_{i}^{*} \omega_{i}^{*}$, this is equivalent to the assertion 


$$
\delta\left(\mathfrak{S}_{p}^{*}\right)=\frac{1}{2} \sum_{i=1}^{r} d_{i} v_{i}^{*}\left(\mathfrak{S}_{i}^{*} \omega_{i}^{*}\right),
$$

which we proceed to verify.

Let $\mathrm{D}^{*}=\sum_{i=1}^{r} \mathrm{D}_{i}^{*} \omega_{i}^{*}$. Then clearly

$$
\delta\left(\mathfrak{C}_{\mathbb{p}}^{*}\right)=\operatorname{dim} \mathfrak{D}_{p}^{*} / \mathfrak{D}^{*}+\sum_{i=1}^{r} \delta\left(\mathfrak{C}_{i}^{*}\right) .
$$

(7) follows at once from (8) and from the following two statements:

$$
\begin{aligned}
& \operatorname{dim} o_{p}^{*} / \mathrm{o}^{*}=\frac{1}{2} \sum_{i=1}^{r} d_{i} v_{i}^{*}\left(\omega_{i}^{*}\right) \\
& \delta\left(\mathfrak{S}_{i}^{*}\right)=\frac{1}{2} d_{i} v_{i}^{*}\left(\mathfrak{S}_{i}^{*}\right), \quad i=1,2, \cdots, r .
\end{aligned}
$$

For each $j, 2 \leqq j \leqq r$, define $\Omega_{i j}^{*}=\prod_{m=1, m \neq i}^{1} F_{m}^{*}, i=1,2, \cdots, j$, and let $\omega_{i j}^{*}$ be the residue of $\Omega_{i j}^{*}$ in $\mathfrak{o}_{i}{ }^{*}$. Also denote by $\mathfrak{Q}_{j}^{*}$ the ideal $\left(\Omega_{1 j}^{*}, \Omega_{2 j}^{*}, \cdots, \Omega_{j j}^{*}\right)$ in $D_{\mathfrak{P}^{*}}$. We shall prove

$$
\operatorname{dim} \mathfrak{D}_{\mathfrak{*}}^{*} / \mathfrak{Q}_{j}^{*}=\frac{1}{2} \sum_{i=1}^{j} d_{i} \boldsymbol{v}_{i}^{*}\left(\omega_{i}{ }^{*}\right) .
$$

Since $\mathfrak{D}_{\mathbf{r}}^{*}=0^{*},\left(9^{\prime}\right)$ implies $(9)$.

We shall prove $\left(9^{\prime}\right)$ by induction on $r$. Let $f_{i}^{*}$ be the residue of $F_{i}^{*}$ in $\mathrm{o}_{i}^{*}, i=1,2, \cdots, r$. If $r=2, \mathfrak{Q}_{2}^{*}=\left(F_{1}^{*}, F_{2}^{*}\right), \omega_{12}^{*}=f_{2}^{*}, \omega_{22}^{*}=f_{1}^{*}$, and $\left(9^{\prime}\right)$ is a direct result of the following lemma:

Lemma 3. $\operatorname{dim} \mathfrak{D}_{\mathfrak{B}}^{*} /\left(F_{1}^{*}, F_{2}^{*}\right)=d_{1} v_{1}^{*}\left(f_{2}^{*}\right)=d_{2} v_{2}^{*}\left(f_{1}^{*}\right)$.

Proof. It suffices to prove the first equality. Since $F_{2}{ }^{*}$ is a nonunit in $\mathfrak{D}_{\mathfrak{B}}^{*}, f_{2}^{*}$ is a nonunit in $\mathfrak{o}_{1}^{*}$. Hence $\mathfrak{D}_{1}^{*}$ contains the ring $T^{*}$, which is defined, according as $K$ is an algebraic number field or an algebraic function field, as the completion of $I\left[f_{2}^{*}\right]$ or of $k\left[f_{2}^{*}\right]$ with respect to the principal ideal $\left(f_{2}{ }^{*}\right)$. $\mathrm{D}_{1}^{*}$ is, in fact, a finite $T^{*}$-module (Chevalley [1, Proposition 5, p. 699]): $\mathrm{o}_{1}^{*}=\sum_{i=1}^{s} T^{*} u_{i}, u_{i} \in \mathrm{o}_{1}^{*}, i=1,2, \cdots, s$. If $L^{*}$ denotes the quotient field of $T^{*}$, it follows that $\left[\Sigma_{1}^{*}: L^{*}\right]=s$. On the other hand, $\left[\Sigma_{1}^{*}: L^{*}\right]=d_{1} v_{1}^{*}\left(f_{2}^{*}\right)$ (Chevalley [2, Theorem 5, p. 61]), whence $s=d_{1} v_{1}^{*}\left(f_{2}^{*}\right)$. Since clearly $\operatorname{dim} \mathfrak{D}_{\mathfrak{B}}^{*} /\left(F_{1}^{*}, F_{2}^{*}\right)=\operatorname{dim}{\mathfrak{o}_{1}}^{*} / \mathfrak{o}_{1}^{*} f_{2}^{*}$, we have only to verify that $\operatorname{dim}{\mathfrak{o}_{1}}^{*} / \mathfrak{o}_{1}^{*} f_{2}^{*}=s$.

If $z \in \mathrm{o}_{1}^{*}, z=\sum_{i=1}^{s} t_{i}^{*} u_{i}, t_{i}^{*} \in T^{*}$. There exist constants $c_{i}$ in $k$ (or $I$ ) such that $t_{i}^{*}-c_{i} \in T^{*} f_{2}^{*}, i=1,2, \cdots, s$. It follows at once that $z-\sum_{i=1}^{s} c_{i} u_{i}$ $\in \mathrm{o}_{1}^{*} f_{2}^{*}$, and hence that $u_{1}, u_{2}, \cdots, u_{s}$ span ${o_{1}}^{*}$ modulo $0_{1}^{*} f_{2}^{*}$. On the other hand, if $\sum_{i=1}^{s} c_{i} u_{i}=z f_{2}{ }^{*}$ with $c_{i} \in k$ (or $I$ ) and $z \in 0_{1}{ }^{*}$, we may write $z=\sum_{i=1}^{s} t_{i}^{*} u_{i}, t_{i}^{*} \in T^{*}$, whence $\sum_{i=1}^{s}\left[c_{i}-f_{2}^{*} t_{i}^{*}\right] u_{i}=0$. Since the $u_{i}$ are linearly independent modulo $T^{*}$, we conclude that $c_{i}=0, i=1,2, \cdots, s$, and so the 
$u_{i}$ are linearly independent modulo $\mathrm{o}_{1}{ }^{*} f_{2}{ }^{*}$. This completes the proof of the lemma.

Now assume $\left(9^{\prime}\right)$ for $j<r$. To prove the assertion for $j=r$, write $\operatorname{dim} \mathfrak{D}_{\mathfrak{Q}}^{*} / \mathfrak{Q}_{r}^{*}=\operatorname{dim} \mathfrak{D}_{\mathfrak{B}}^{*} / \mathfrak{Q}_{r-1}^{*}+\operatorname{dim} \mathfrak{Q}_{r-1}^{*} / \mathfrak{Q}_{r}^{*}$, apply the induction hypothesis for $j=r-1$, and use the equality

$$
\operatorname{dim}\left(\mathfrak{Q}_{r-1}^{*} / \mathfrak{Q}_{r}^{*}\right)=\sum_{i=1}^{r-1} d_{r} \boldsymbol{v}_{r}^{*}\left(f_{i}^{*}\right),
$$

to be verified in the next paragraph. A direct computation will yield $\left(9^{\prime}\right)$ at once.

Define the ideals $\mathfrak{S}_{h}^{*}$ in $\mathfrak{D}_{\mathfrak{B}}^{*}, h=0,1, \cdots, r-1$, as follows: $\mathfrak{S}_{0}^{*}$ $=\sum_{e=1}^{r-1}\left(\Omega_{e r-1}^{*}\right) ; \mathfrak{S}_{h}^{*}=\sum_{e=1}^{h} \Omega_{e r-1}^{*}\left(F_{e}^{*}, F_{r}^{*}\right)+\sum_{e=h+1}^{r-1}\left(\Omega_{e r-1}^{*}\right), h=1,2, \cdots$, $r-1$. Clearly $\mathfrak{S}_{0}^{*}=\mathfrak{Q}_{r-1}^{*}, \mathfrak{S}_{r-1}^{*}=\mathfrak{Q}_{r}^{*}$. Since $\mathfrak{S}_{h-1}^{*} \supset \mathfrak{S}_{h}^{*}$, we prove (11) by showing that $\operatorname{dim}\left(\mathfrak{S}_{h}^{*} / \mathfrak{S}_{h-1}^{*}\right)=d_{r} v_{r}^{*}\left(f_{h}^{*}\right), h=1,2, \cdots, r-1$. In fact, if $A_{i}^{*}$, $i=1,2, \cdots, d_{r} v_{r}^{*}\left(f_{h}^{*}\right)$, form a basis for $\mathfrak{D}_{\mathfrak{B}}^{*} \operatorname{modulo}\left(F_{h}^{*}, F_{r}^{*}\right)$, it follows from the definition of $\mathfrak{S}_{h}^{*}$ that the elements $A_{i}^{*} \Omega_{h r-1}^{*}$ span $\mathfrak{S}_{h-1}^{*}$ modulo $\mathfrak{S}_{h}^{*}$. Moreover, if $A^{*} \in \bigcup_{\mathfrak{B}}^{*}$ and $A^{*} \Omega_{h r-1}^{*} \in \mathfrak{S}_{h}^{*}$, it follows easily that $A^{*} \Omega_{h r-1}^{*}$ $\equiv B^{*} \Omega_{h r-1}^{*} F_{r}^{*}\left(\bmod F_{h}^{*}\right), B^{*} \in \bigvee_{\mathfrak{P}}^{*}$. Since $\Omega_{h r-1}^{*}$ and $F_{h}^{*}$ are relatively prime, we have $A^{*} \in\left(F_{h}^{*}, F_{r}^{*}\right)$, which implies that the elements $A_{i}{ }^{*} \Omega_{h r-1}^{*}$ are linearly independent modulo $\mathfrak{S}_{h}^{*}$. Hence $\operatorname{dim}\left(\mathfrak{S}_{h-1}^{*} / \mathfrak{S}_{h}^{*}\right)=d_{r} v_{r}^{*}\left(f_{h}^{*}\right)$, as asserted.

To prove $(10)$, let $\mathfrak{B}_{i}$ be the valuation ideal in $\mathfrak{D}$ defined by $\boldsymbol{v}_{i}\left(\mathfrak{B}_{i}\right)$ $=\boldsymbol{v}_{i}{ }^{*}\left(\mathfrak{S}_{i}^{*}\right)$. In view of the natural correspondence of the $\boldsymbol{v}_{i}{ }^{*}$-ideals in $\boldsymbol{o}_{i}{ }^{*}$ with $v_{i}$-ideals in $\mathfrak{D}$, we have only to prove $\delta\left(\mathfrak{B}_{i}\right)=2^{-1} d_{i} v_{i}\left(\mathfrak{B}_{i}\right), i=1,2, \cdots, r$.

Theorem 1 implies that $\mathfrak{B}_{i}$ is the largest regular $v_{i}$-ideal in $\mathfrak{D}$. For definiteness, take $i=1$, and let $\left\{\mathfrak{q}_{i}\right\}$ be the sequence of $v_{1}$-ideals in $\mathbf{D}$. Let $\pi_{1}, \cdots, \pi_{n}$ be the set of elements, constructed in Theorem 5 for $v_{1}$, and let $d^{\prime}=\prod_{i=2}^{n} s_{i}$. Let $q_{h}$ be the $v_{1}$-ideal in the sequence of value $\nu_{d^{\prime}}\left(b_{1}, \cdots, b_{n}\right)$. Theorem 4 (a) implies that, for all $j \geqq h$, and for all $m \geqq 0, v_{1}\left(\mathfrak{q}_{j+m}\right) \in R_{d^{\prime}}\left(b_{1}, \cdots, b_{n}\right)$, and hence that there exist $d_{1}=d_{\mathfrak{p}} d^{\prime}$ distinct canonical products of $\pi_{1}, \cdots, \pi_{n}$ of value $\boldsymbol{v}_{\mathbf{1}}\left(\mathfrak{q}_{j+m}\right)$. Since these canonical products are linearly independent modulo $\mathfrak{q}_{j+m+1}$, we conclude that $q_{j}$ is a regular $v_{1}$-ideal for all $j \geqq h$.

On the other hand, $\nu_{d^{\prime}}\left(b_{1}, \cdots, b_{n}\right)-1 \in R_{d^{\prime}-1}\left(b_{1}, \cdots, b_{n}\right)$, by Theorem 4 (b), whence there exist only $d_{\mathfrak{p}}\left(d^{\prime}-1\right)<d_{1}$ canonical products of $\pi_{1}, \cdots, \pi_{n}$ of value $v_{1}\left(\mathfrak{q}_{h-1}\right)$ which are linearly independent modulo $\mathfrak{q}_{h}$. It follows that neither is $\mathfrak{q}_{h-1}$ nor any $\mathfrak{q}_{j} \supset \mathfrak{q}_{h-1}$ a regular $v_{1}$-ideal. We conclude that $\mathfrak{q}_{h}=\mathfrak{B}_{1}$, and consequently $v_{1}\left(\mathfrak{B}_{1}\right)=\nu_{d^{\prime}}\left(b_{1}, \cdots, b_{n}\right)$.

Since $\delta\left(\mathfrak{B}_{1}\right)=\sum_{j=0}^{h-1} \operatorname{dim}\left(\mathfrak{q}_{j} / \mathfrak{q}_{j+1}\right)$, where $\mathfrak{q}_{0}=\mathfrak{b}$, and since $\operatorname{dim}\left(\mathfrak{q}_{j} / \mathfrak{q}_{j+1}\right)$ $=d_{p} \chi\left(v_{1}\left(\mathfrak{q}_{j}\right)\right)$, it follows that $\delta\left(\mathfrak{B}_{1}\right)=d_{\mathfrak{p}} \chi \sum_{j=0}^{n-1}\left(v_{1}\left(\mathfrak{q}_{j}\right)\right)$. Furthermore by Theorem 4 (c) we have $\sum_{j=0}^{h-1} \chi\left(v_{1}\left(q_{j}\right)\right)=2^{-1} d^{\prime} \nu_{d^{\prime}}\left(b_{1}, \cdots, b_{n}\right)$, whence $\delta\left(\mathfrak{B}_{1}\right)$ $=2^{-1} d_{1} v_{1}\left(\mathfrak{B}_{1}\right)$.

CoROLlaRY. If $\overline{\mathfrak{D}}$ is the integral closure of $\mathfrak{D}$ in $K$, and $\mathfrak{S}$ is the conductor between $\overline{\mathrm{D}}$ and $\mathrm{D}$, then $\operatorname{dim}(\overline{\mathrm{D}} / \mathfrak{\mathbb { S }})=2 \operatorname{dim}(\mathrm{o} / \mathfrak{S})$. 
Proof. Let $\mathfrak{p}$ be a prime ideal in $\mathfrak{o}$. Clearly $\mathfrak{o}_{\mathfrak{p}} \mathbb{C} \subset \mathfrak{C}_{\mathfrak{p}}$. Since $\overline{\mathfrak{o}}_{\mathfrak{p}}$ is a finite $\mathfrak{o}_{\mathrm{p}}$-module (compare Chevalley [2, Theorem 3, chap. 4] or Zariski [12, p. 507]), we have $\overline{\mathfrak{D}}_{\mathfrak{p}}=\sum_{i=1}^{m} \mathfrak{o}_{\mathfrak{p}} \beta_{i}, \beta_{i} \in K, i=1,2, \cdots, m$. If $z \in \mathfrak{C}_{\mathfrak{p}}$, then $z \beta_{i} \in \mathfrak{o}_{\mathfrak{p}}$, for all $i=1,2, \cdots, m$. Write $z \beta_{i}=\left(\eta_{i} / \eta_{0}\right), \eta_{i} \in K, i=0,1, \cdots, m$, and $\eta_{0} \notin \mathfrak{p}$. Then $\left(\eta_{0} z\right) \beta_{i}=\eta_{i} \in \mathfrak{0}$ for each $i$, whence $\eta_{0} z \in \mathbb{C}$. Hence $z \in \mathfrak{o}_{\mathfrak{p}} \mathbb{E}$, and it follows that $\mathbb{D}_{\mathfrak{p}} \mathfrak{E}=\mathfrak{C}_{\mathrm{p}}$.

Let $\mathbb{E}=\bigcap_{i=1}^{s} q_{i}$ be a representation of $\mathbb{E}$ as the intersection of primary ideals, and let $\mathfrak{p}_{i}$ be the associated prime ideal of $\mathfrak{q}_{i}$. The preceding equality implies that $\mathfrak{q}_{i}=\mathfrak{G}_{\mathfrak{p}_{i}} \cap \mathbb{0}, i=1,2, \cdots, s$, and, since $\mathfrak{C}_{\mathfrak{p}_{i}}$ is a complete $\mathfrak{p}_{i}$-ideal in $\mathfrak{D}_{\mathfrak{i}}$, that each $\mathfrak{q}_{i}$ is a complete $\mathfrak{p}_{i}$-ideal in $\mathfrak{o}$. Hence $\mathfrak{E}$ is a complete ideal in $\mathfrak{o}$, and $d(\mathfrak{C})=\operatorname{dim}(\overline{\mathfrak{o}} / \mathfrak{C})=\sum_{i=1}^{s} d\left(\mathfrak{C}_{\mathfrak{p}_{i}}\right)$. Moreover, it is readily verified that $\delta(\mathfrak{C})=\operatorname{dim}(\mathfrak{o} / \mathbb{C})=\sum_{i=1}^{s} \delta\left(\mathfrak{C}_{\mathfrak{p}_{i}}\right)$. The corollary now follows at once from the theorem.

\section{Part II. The Geometry IN The LARGE}

6. Geometric preliminaries. Throughout part II, we consider a fixed irreducible plane curve $\Gamma: F\left(X_{0}, X_{1}, X_{2}\right)=0$ of degree $m$, defined over an arbitrary ground field $k$, with function field $K$. We denote by $\left(x_{0}, x_{1}, x_{2}\right)$ the set of homogeneous coordinates of $\Gamma$. For our purposes, it will be convenient to define $a$ point of $\Gamma$ to be a complete set of conjugate points in the ordinary sense. It will also help to make a few minor changes in the notation of part I. If $P$ is a point of $\Gamma$, we denote by $\mathfrak{o}_{P}$ (rather than $\mathfrak{o}_{\mathfrak{p}}$ ) the local ring of $P$ on $\Gamma$. If $v_{i}, i=1,2, \cdots, r$, are the valuations of $K$ whose valuation rings contain $\mathfrak{D}_{P}$, we say that each $\boldsymbol{v}_{\boldsymbol{i}}$ has center $P$ on $\Gamma$, and call the intersection of $\boldsymbol{v}_{\boldsymbol{i}}$-ideals, $i=1,2, \cdots, r$, in $\mathfrak{o}_{P}$ a complete $P$-ideal. Because of the identification of conjugate points, every valuation of $K$ has a uniquely determined center on $\Gamma$.

By definition, a prime divisor of $K$ is a complete set of conjugate places of $K$. To each prime divisor $p$ is associated a unique valuation $v_{p}$ of $K$, and conversely. By the center of $\boldsymbol{p}$ on $\Gamma$ is meant the center of the corresponding valuation $\boldsymbol{v}_{\boldsymbol{p}}$, and by the degree $d(\boldsymbol{p})$ of $\boldsymbol{p}$ is meant $d\left(\boldsymbol{v}_{\boldsymbol{p}}\right)$. A divisor of $K$ is any formal power product $B=p_{1}^{\mu_{1}} p_{2}^{\mu_{2}} \cdots p_{s}^{\mu_{s}}$ of prime divisors $p_{1}, \cdots, p_{s}$ with arbitrary integral exponents $\mu_{1}, \cdots, \mu_{s}$. Its degree $d(B)$ is defined to be $d(\boldsymbol{B})=\sum_{i=1}^{s} \kappa_{i} d\left(\boldsymbol{p}_{i}\right)$.

Let $\Gamma^{\prime}: G\left(X_{0}, X_{1}, X_{2}\right)=0$ be a curve not containing $\Gamma$ as a component, and let $p$ be a prime divisor of $K$. If $P$ is the center of $p$ on $\Gamma, X_{\lambda} \neq 0$ at $P$ for some $\lambda=0,1$, or 2 . We define the intersection of $\Gamma$ and $\Gamma^{\prime}$ at $p$ to be the divisor $p^{\mu}$ where $\mu=v_{p}\left(G\left(\left(x_{0} / x_{\lambda}\right),\left(x_{1} / x_{\lambda}\right),\left(x_{2} / x_{\lambda}\right)\right)\right)$, and define the intersection multiplicity $i\left(p ; \Gamma, \Gamma^{\prime}\right)$ of $\Gamma$ and $\Gamma^{\prime}$ at $p$ to be $d\left(p^{\mu}\right)$. If $p_{1}=p, p_{2}, \cdots, p_{r}$ are all the prime divisors of center $P$ on $\Gamma$, we define the intersection multiplicity $i\left(P ; \Gamma, \Gamma^{\prime}\right)$ to be $\sum_{j=1}^{r} i\left(p_{j} ; \Gamma, \Gamma^{\prime}\right)$. Thus the complete intersection of $\Gamma$ and $\Gamma^{\prime}$ is a divisor $\left(\Gamma, \Gamma^{\prime}\right)$ whose degree $i\left(\Gamma, \Gamma^{\prime}\right)=\sum_{P \in \Gamma} i\left(P ; \Gamma, \Gamma^{\prime}\right)$. Bézout's theorem asserts that if $\Gamma^{\prime}$ is of degree $n, \boldsymbol{i}\left(\Gamma, \Gamma^{\prime}\right)=m \cdot n$.

It will be convenient to denote by $g(n)$ the linear series cut out on $\Gamma$ by 
the system of all curves of order $n$, and by $g(B ; n)$ the system of all curves of order $n$ whose complete intersection with $\Gamma$ contains the integral divisor $B$ as a fixed component. The quantity $\operatorname{dim} g(n)-\operatorname{dim} g(B ; n)$ is a constant for all $n$ sufficiently large. We define $\delta(B)=\operatorname{dim} g(n)-\operatorname{dim} g(B ; n)$ for large $n$. $\delta(B)$ designates the number of conditions which the divisor $B$ imposes on the curves of sufficiently high degree.

Let $B_{i}, i=1,2, \cdots, h$, be integral divisors, and assume that each prime factor of $B_{i}$ has its center at a given point $P_{i}$ of $\Gamma, P_{i} \neq P_{j}, i \neq j$. Then the theorem on the independence of conditions asserts that, if $B=\prod_{i=1}^{h} B_{i}$, then

$$
\delta(\boldsymbol{B})=\sum_{i=1}^{h} \delta\left(\boldsymbol{B}_{i}\right) .
$$

Let $P$ be a point of $\Gamma$ and let $p_{1}, \cdots, p_{r}$ be the prime divisors of $K$ of center $P$, with $v_{1}, \cdots, v_{r}$ the corresponding valuations. There is a natural one-one correspondence between the integral divisors of the form $A$ $=p_{1}^{\mu_{1}} \cdot \cdots p_{\tau}^{\mu_{r}}$ and complete $P$-ideals $\mathfrak{q}$ in $\mathfrak{D}_{P}: A \leftrightarrow \mathfrak{q}$ if and only if $\mu_{i}=v_{i}(\mathfrak{q})$, $i=1,2, \cdots, r$. For corresponding pairs $A, \mathfrak{q}$, the

$$
\text { basic equalities } \delta(\boldsymbol{A})=\delta(\mathfrak{q}), \quad d(\boldsymbol{A})=d(\mathfrak{q})
$$

are easily verified.

7. Adjoint curves. Let $P$ be a point of $\Gamma$. The conductor $\mho_{P}$ between $\mathfrak{D}_{P}$ and $\overline{\mathfrak{D}}_{P}$ is, by Theorem 3 , a complete $P$-ideal. The divisor corresponding to $\mathfrak{C}_{P}$ is designated by $\boldsymbol{C}_{P}$ and is called the adjoint divisor at $P$.

If $P$ is a simple point of $\Gamma, C_{P}$ is the unit divisor. Since $\Gamma$ has only a finite number of singular points, the divisor $\boldsymbol{C}=\prod_{P \in \mathrm{r}} \boldsymbol{C}_{P}$ is well-defined and is called the adjoint divisor of $\Gamma$.

Definition. An adjoint curve to $\Gamma$ is any curve which cuts out on $\Gamma$ a multiple of the adjoint divisor $\boldsymbol{C}$.

LemмA 4. If $\boldsymbol{A}$ is any integral divisor, we have $\delta(\boldsymbol{A C})=\delta(\boldsymbol{C})+d(\boldsymbol{A})$.

Proof. Let $\boldsymbol{A C}=\prod_{i=1}^{t} A_{P_{i}} C_{P_{i}}$ where $A_{P_{i}}, C_{P_{i}}$ are the factors of $A$ and $C$ respectively which contain those prime divisors of center $P_{i}$ on $\Gamma$. Let $q_{i}$ be the complete $P_{i}$-ideal in $\mathfrak{D}_{P_{i}}$ which corresponds to $A_{P_{i}} C_{P_{i}}, i=1,2, \cdots, t$. It follows at once from equations (12) and (13) that $\delta(\boldsymbol{C})=\sum_{i=1}^{t} \delta\left(\boldsymbol{C}_{P_{i}}\right)$ $=\sum_{i=1}^{t} \delta\left(\mathfrak{S}_{P_{i}}\right)$, and $\delta(A C)=\sum_{t=1}^{t} \delta\left(A_{P_{i}} C_{P_{i}}\right)=\sum_{i=1}^{t} \delta\left(\mathfrak{q}_{i}\right)$, whence $\delta(A C)$ $=\delta(\boldsymbol{C})+\sum_{i=1}^{t}\left[\delta\left(\mathfrak{q}_{i}\right)-\delta\left(\mathfrak{S}_{P_{i}}\right)\right]$. Since each $\mathfrak{S}_{P_{i}}$ is a regular ideal $\left(^{4}\right)$,

(4) In the case in which $k$ is infinite a simple proof of the regularity of $\mathfrak{E}_{P}$, for any point $P$ of $\Gamma$, can be given. For then there exists an element $z \in \mathfrak{C}_{P}$ such that $v_{j}(z)=v_{j}\left(\mathfrak{S}_{P}\right), j=1,2, \cdots, r$, where $v_{1}, v_{2}, \cdots, v_{r}$ are valuations of $K$ of center $P$ on $\Gamma$. Let $q \subset \mathbb{E}_{P}$ be a complete $P$-ideal. Define the ideal $q^{\prime}$ in $\overline{\mathbf{D}}_{P}$ by the relations: $v_{j}\left(\mathfrak{q}^{\prime}\right)=v_{j}(\mathfrak{q})-v_{j}\left(\mathbb{E}_{P}\right), j=1,2, \cdots, r$. Then $\operatorname{dim} \bar{v}_{P} / q^{\prime}$ $=d(\mathfrak{q})-d\left(\mathfrak{\complement}_{P}\right)$. If $\omega_{i}, i=1,2, \cdots, d(\mathfrak{q})-d\left(\mathfrak{S}_{P}\right)$, form an independent basis for $\overline{\mathbf{D}}_{P} \bmod \mathfrak{q}^{\prime}$, the elements $z \omega_{i}$ form an independent basis for $\mathfrak{E}_{P} \bmod \mathfrak{q}$. Thus $\operatorname{dim} \mathfrak{C}_{P} / \mathfrak{q}=d(\mathfrak{q})-d\left(\mathfrak{C}_{P}\right)$ and so $\mathfrak{C}_{P}$ is a regular ideal. 


$$
\delta\left(\mathfrak{q}_{i}\right)-\delta\left(\mathfrak{G}_{P_{i}}\right)=d\left(\mathfrak{q}_{i}\right)-d\left(\mathfrak{G}_{P_{i}}\right)=d\left(A_{P_{i}}\right) .
$$

Hence $\delta(A C)=\delta(C)+d(A)$, as asserted.

Theorem 7 (The Noether Fundamentalsatz). Let $\Gamma^{\prime}: G(X)=0$ be $a$ curve not containing $\Gamma$ as a component, and let $A=\left(\Gamma, \Gamma^{\prime}\right)$. Then if $\Gamma^{\prime \prime}: H(X)=0$ cuts out a multiple of $A C$ on $\Gamma$, there exist forms $A(X), B(X)$ with coefficients in $k$ such that $H(X)=A(X) F(X)+B(X) G(X)$.

Proof $\left(^{5}\right)$. We treat first the case in which the degree $n$ of $\Gamma^{\prime \prime}$ is sufficiently high so that $\delta(\boldsymbol{A C})=\operatorname{dim} g(n)-\operatorname{dim}(\boldsymbol{A C} ; n)$, and also that $n \geqq m+e$, where $e$ is the degree of $\Gamma^{\prime}$. In this case,

$$
\operatorname{dim} g(A C ; n)=(n+1)(n+2) / 2-(n-m+1)(n-m+2) / 2-\delta(A C) .
$$

Since $\delta(\boldsymbol{A C})=\delta(\boldsymbol{C})+d(\boldsymbol{A})$ by Lemma 4 and since $d(\boldsymbol{A})=m \cdot e$, it follows that

$$
\operatorname{dim} g(\boldsymbol{A C} ; n)=n m-e m-(1 / 2) m(m-3) / 2-\delta(\boldsymbol{C}) .
$$

On the other hand, let $g^{\prime}$ denote the linear series cut out on $\Gamma$ by the system of all curves of the form $A(X) F(X)+B(X) G(X)=0$ of degree $n$ for which $B(X)=0$ is an adjoint curve. Clearly $g^{\prime} \subset g(A C ; n)$, and $\operatorname{dim} g^{\prime} \geqq \operatorname{dim} g(\boldsymbol{C} ; n-m)=n m-e m\left(m^{2}-3 m\right) / 2-\delta(\boldsymbol{C})$. It follows from (14) that $g^{\prime}=g(A C ; n)$.

If the degree $n$ of $\Gamma^{\prime \prime}$ is arbitrary, let $P_{1}, \cdots, P_{s}$ be the points of intersection of $\Gamma$ and $\Gamma^{\prime}$. There exists an irreducible curve $\phi(X)=0$ which does not pass through any $P_{i}, i=1,2, \cdots, s$. By the first part of the theorem, we have, for $\sigma$ sufficiently large,

$$
\phi(X)^{\sigma} H(X)=A(X) F(X)+B(X) G(X),
$$

for suitable forms $A(X), B(X)$. Let $X=\left(X_{1} / X_{0}\right), Y=\left(X_{2} / X_{0}\right)$, and let $\xi_{1}, \xi_{2}$ be the residues of $X, Y$ respectively modulo $\phi(1, X, Y)$. Let $\left(G\left(1, \xi_{1}, \xi_{2}\right)\right)$ $=\bigcap_{j=1}^{h} q_{j}$ be the decomposition of this ideal into primary components in the ring $k\left[\xi_{1}, \xi_{2}\right]$. Since $A\left(1, \xi_{1}, \xi_{2}\right) F\left(1, \xi_{1}, \xi_{2}\right)=-B\left(1, \xi_{1}, \xi_{2}\right) G\left(1, \xi_{1}, \xi_{2}\right)$, $A\left(1, \xi_{1}, \xi_{2}\right) F\left(1, \xi_{1}, \xi_{2}\right) \in q_{j}$ for all $j=1,2, \cdots, h$. If, for some $j, F\left(1, \xi_{1}, \xi_{2}\right)$ $\in \operatorname{rad} q_{j}$, it would imply that the three curves $\Gamma, \Gamma^{\prime}$, and $\phi(X)=0$ have a point in common, contrary to the construction of $\phi(X)$. Hence $A\left(1, \xi_{1}, \xi_{2}\right)$ $\in \bigcap_{j=1}^{h} \mathfrak{q}_{j}=\left(G\left(1, \xi_{1}, \xi_{2}\right)\right)$. It follows easily that $A(X)=A_{0}(X) G(X)+A_{1}(X) \phi(X)$, $B(X)=-A_{0}\left(X_{0}\right) G(X)+B_{1}(X) \phi(X)$ for suitable forms $A_{0}(X), A_{1}(X)$, and $B_{1}(X)$.

Substituting these relations in (15), we conclude that $\phi(X)^{\sigma-1} H(X)$ $=A_{1}(X) F(X)+B_{1}(X) G(X)$. By $\sigma$ repetitions of this argument, we complete the proof of the theorem.

The completeness of the adjoint series is an immediate corollary of the

(5) Compare the proof in Severi [9]. 
Noether Fundamentalsatz, as is well known $\left(^{6}\right)$.

THEOREM 8. The adjoint curves of any order cut out complete linear series on $\Gamma$.

8. Adjoint curves of order $m-3$ and the canonical series $\left({ }^{7}\right)$. Our present object is to derive the following basic properties of the adjoint curves:

THEOREM 9. The adjoint curves of order $m-3$ cut out on $\Gamma$ outside of the fixed component $\boldsymbol{C}$ the complete canonical series.

TheOREM 10. $d(\mathbf{C})=2 \delta(\boldsymbol{C})$.

As a direct corollary of these two results, we shall also obtain:

THEOREM 11. If the genus $\left(^{8}\right)$ of $\Gamma$ is denoted by $g$, then

$$
g=(m-1)(m-2) / 2-\delta(\boldsymbol{C}) .
$$

We shall give two independent proofs of Theorems 9 and 10. In fact, we shall prove first that these two theorems are equivalent to each other. We shall then show that Theorem 10 is a direct consequence of Theorem 6 , the main theorem of part I. Finally we shall give a proof of Theorem 9, in the case in which the function field $K$ of $\Gamma$ is separably generated over $k$, based upon a representation theorem for the differentials of the first kind of $K$.

Assume first that $d(\boldsymbol{C})=2 \delta(\boldsymbol{C})$. Take $n$ so large that $\operatorname{dim} g(n)-\operatorname{dim} g(\boldsymbol{C} ; n)$ $=\delta(\boldsymbol{C})$ and that $g(\boldsymbol{C} ; n)$ is a nonspecial series $\left({ }^{9}\right)$. Then, as in $(14), \operatorname{dim} g(\boldsymbol{C} ; n)$ $=m n-\left(m^{2}-3 m\right) / 2-\delta(\boldsymbol{C})$. On the other hand, since $g(\boldsymbol{C} ; n)$ is a complete series of degree $m n-d(\boldsymbol{C})$, it follows by the Riemann-Roch theorem (Chevalley [2, Theorem 7, p. 33]) that $\operatorname{dim} g(\mathbf{C} ; n)=m n-d(\mathbf{C})-g+1$, whence

$$
(m-1)(m-2) / 2+\delta(\boldsymbol{C})-d(\boldsymbol{C})=g .
$$

Since $d(\boldsymbol{C})=2 \delta(\boldsymbol{C})$, we conclude that

(6) B. L. van der Waerden $[10$, p. 216].

(7) For the definitions of the various concepts concerning algebraic curves which we shall need in this section, we refer the reader to Chapters I and II of Chevalley [2]. We point out, however, that there exists a direct connection between the notion of a divisor class of $K$ as defined by Chevalley on p. 19 and the concept of a complete linear series on $\Gamma$ : namely, the integral divisors in a divisor class of $K$ form a complete linear series, which can be cut out on $\Gamma$ by a linear system of plane curves. In particular, the integral divisors in the canonical divisor class (Chevalley p. 32) form the canonical series. Furthermore the degree and dimension of a divisor class is equal respectively to the degree and dimension of the corresponding complete linear series. Hence the canonical series is of dimension $g$ and of degree $2 g-2$, where $g$ is the genus of $K$ (Chevalley, chapter II, Theorems 4 and 6).

( $\left.{ }^{8}\right)$ By the genus of $\Gamma$ we mean the genus of the function field $K$ of $\Gamma$.

( $\left.{ }^{9}\right)$ A linear series is called special if it is a subseries of the canonical series; otherwise it is called nonspecial. A series of degree greater than $2 g-2$ is necessarily nonspecial (Chevalley $[2$, p. 32, corollary $])$. 


$$
m(m-3)-d(\mathbf{C})=2 g-2,
$$

and

$$
(m-1)(m-2) / 2-\delta(\mathbf{C})=g
$$

(which establishes Theorem 11).

(17) implies that $\operatorname{deg} g(\boldsymbol{C} ; m-3)=2 g-2$, while (18) implies that $\operatorname{dim} g(\boldsymbol{C} ; m-3) \geqq g$. Since $g(\boldsymbol{C} ; m-3)$ is complete, it follows at once from the Riemann-Roch theorem that $g(C ; m-3)$ is a special series. In fact, $\operatorname{dim} g(\boldsymbol{C} ; m-3)-\operatorname{deg} g(\boldsymbol{C} ; m-3)+g-1 \geqq g-(2 g-2)+g-1=1$, whence $g(C ; m-3)$ is a subseries of the canonical series. Since its degree, $2 g-2$, is equal to the degree of the canonical series, it follows that $g(C ; m-3)$ is itself the complete canonical series.

Conversely, if we assume Theorem 9, then

$$
\operatorname{deg} g(\boldsymbol{C} ; m-3)=m(m-3)-d(\mathbf{C})=2 g-2 .
$$

Combining this equality with equation (16) gives at once $d(\boldsymbol{C})=2 \delta(\boldsymbol{C})$.

To prove Theorem 10 , let $P_{1}, P_{2}, \cdots, P_{s}$ be the singular points of $\Gamma$. Since $d\left(\mathfrak{G}_{\boldsymbol{P}_{\boldsymbol{i}}}\right)=2 \delta\left(\mathfrak{G}_{P_{\boldsymbol{i}}}\right), i=1,2, \cdots, s$, by Theorem 6 , we have $d\left(\boldsymbol{C}_{P_{i}}\right)$ $=2 \delta\left(C_{P_{i}}\right), i=1,2, \cdots, s$, in view of (13). Since $C=\prod_{i=1}^{s} C_{P_{i}}$, it follows immediately that $d(\boldsymbol{C})=2 \delta(\boldsymbol{C})$.

Finally let $(X, Y)$ be a set of nonhomogeneous plane coordinates. For simplicity, denote by $F(X, Y)=0$ the nonhomogeneous equation of $\Gamma$, of degree $m$, and let $(x, y)$ be corresponding nonhomogeneous coordinates of the general point of $\Gamma$. Assume that the function field $K=k(x, y)$ of $\Gamma$ is separably generated over $k$. Then either $x$ or $y$ is a separating variable. We assume, for definiteness, that $x$ is a separating variable, and that $y$ is an integral element over $k(x)\left({ }^{10}\right)$.

If $\Gamma^{\prime}: \phi\left(X_{0}, X_{1}, X_{2}\right)=0$ is an adjoint curve to $\Gamma$, we shall write $\phi(X, Y)=0$ for the nonhomogeneous equation of $\Gamma^{\prime}$, and shall say that $\phi(X, Y)=0$ is an adjoint curve of order $n$ if $\operatorname{deg} \phi(X, Y) \leqq n$.

Since the differentials of $K\left({ }^{11}\right)$ form one complete divisor class-the canonical class-Theorem 9 is a direct corollary of the following representation theorem.

Theorem 12. A differential $\tilde{\omega}$ of $K$ is of the first kind if and only if it can be written in the form $\left(\phi(x, y) / F_{y}^{\prime}(x, y)\right) d x$, where $\Gamma^{\prime}: \phi(X, Y)=0$ is an adjoint curve of order $m-3$. If $\tilde{\omega}$ is of the first kind, the divisor of $\tilde{\omega}=\left(\Gamma, \Gamma^{\prime}\right) / C$.

(10) If $k$ is infinite, this is no restriction; for then $a x+b y=y_{1}$ will be integral over $k(x)$ for suitable $a, b \in k$, and the curve $\Gamma_{1}$ whose general point is $x, y_{1}$ will be projectively equivalent to $\Gamma$.

(11) For the definitions of a differential of $K$, the order of a differential, the divisor of a differential, a differential of the first kind, etc., see Chevalley [2, chapter II, $\$ \$ 5$ and 6]. Since $x$ is a separating variable for $K$, every differential of $K$ is of the form $A d x, A \in K$ (Chevalley. chapter VI, Theorem 4). 
Proof. If $\tilde{\omega}$ is any differential of $K$, we can write $\tilde{\omega}=\left(\phi(x, y) / F_{y}^{\prime}(x, y)\right) d x$, where $\phi(x, y) \in K$. Let $\mathfrak{D}=k[x, y]$, and let $\mathfrak{S}$ be the conductor between $\overline{\mathfrak{D}}$ and D. The following results are well known:

$\mathfrak{b} F_{y}^{\prime}(x, y)=\mathfrak{S} \mathfrak{D}$ where $\mathfrak{D}$ is the different of $K$ with respect to $k(x)$ (Hecke[6, p. 145]).

$v_{p}(\mathfrak{D})=v_{p}(d x)$ for any prime divisor $\boldsymbol{p}$ of $K$ whose center on $\Gamma$ is at finite distance in the $(X, Y)$-plane (Chevalley [2, Theorem 7, p. 110]).

Together these results imply that $\boldsymbol{v}_{p}(\tilde{\omega}) \geqq 0$ if and only if $\boldsymbol{v}_{p}(\phi(x, y))$ $\geqq v_{p}(\mathbb{S})$. Thus $\tilde{\omega}$ is of the first kind at finite distance if and only if $v_{p}(\phi(x, y))$ $\geqq v_{p}(\mathbb{E})$ for all prime divisors of center at finite distance on $\Gamma$. Since $\mathbb{C}$ is a complete ideal, this last condition is equivalent to the assertion $\phi(x, y)$ $\in \mathbb{S}$, which in turn is equivalent to the statements: $\phi(x, y)$ is a polynomial in $x, y$ and $\phi(x, y) \in \mathfrak{S}_{P}$ for every point $P$ of $\Gamma$ at finite distance (compare proof of corollary to Theorem 6).

Consider now the nonhomogeneous plane coordinates: $X^{\prime}=X_{0} / X_{1}$, $Y^{\prime}=X_{2} / X_{1}$; and $\bar{X}=X_{0} / X_{2}, \bar{Y}=X_{1} / X_{2}$. Every point $P$ of $\Gamma$ at infinity in the $(X, Y)$-plane (at least one such point exists) is at finite distance in either the $\left(X^{\prime}, Y^{\prime}\right)$ - or $(\bar{X}, \bar{Y})$-plane.

If $x^{\prime}, y^{\prime}$ are nonhomogeneous $\left(X^{\prime}, Y^{\prime}\right)$-coordinates of $\Gamma$, then $x^{\prime}=1 / x$, $y^{\prime}=y / x$. Write $F(X, Y)=F^{\prime}\left(X^{\prime}, Y^{\prime}\right) / X^{\prime m}$ and $\phi(X, Y)=\phi^{\prime}\left(X, Y^{\prime}\right) / X^{\prime h}$, where $h$ is the degree of $\phi(X, Y)$. We have $F_{y}^{\prime}(x, y)=F_{y^{\prime}}^{\prime}\left(x^{\prime}, y^{\prime}\right) / x^{\prime m-1}$ and also, since $x$ is a separating variable, $d x=-\left(1 / x^{\prime 2}\right) d x^{\prime}$. It follows that $\tilde{\omega}=-\left(\phi^{\prime}\left(x^{\prime}, y^{\prime}\right) x^{\prime m-3-h} / F^{\prime}{ }_{y^{\prime}}\left(x^{\prime}, y^{\prime}\right)\right) d x^{\prime}$.

In the second case, write $F(X, Y)=\bar{F}(\bar{X}, \bar{Y}) / \bar{Y}^{m}, \phi(X, Y)=\bar{\phi}(\bar{X}, \bar{Y}) / \bar{Y}^{h}$, and let $\bar{x}, \bar{y}$ be nonhomogeneous $(\bar{X}, \bar{Y})$-coordinates of $\Gamma$. Then $\bar{x}=x / y$, $\bar{y}=1 / y$. If $y$ is also a separating variable, then $d x / F_{y}^{\prime}(x, y)=-d y / F_{x}^{\prime}(x, y)$, and it follows that $\tilde{\omega}=-\left(\bar{\phi}(x, y) \bar{y}^{m-3-h} / \bar{F}_{\bar{y}}^{\prime}(\bar{x}, \bar{y})\right) d \bar{x}$. If, however, $y$ is not a separating variable, we find directly that $F_{y}^{\prime}(x, y)=\bar{F}_{y}^{\prime}(\bar{x}, \bar{y}) / \bar{y}^{m-2}$. Let $D_{x}$ be the unique derivation of $K$ for which $D_{x}(x)=1$. We have then by Chevalley [2, Chap. 6, Theorem 9] $d \bar{x}=D_{x}(\bar{x}) d x=\left(1 / y-\left(x / y^{2}\right) D_{x}(y)\right) d x=(1 / y) d x$ since $D_{x}(y)=0$. We conclude in this case that $\tilde{\omega}=\left(\bar{\phi}(\bar{x}, \bar{y}) \bar{y}^{m-3-h} / \bar{F}_{\bar{y}}^{\prime}(\bar{x}, \bar{y})\right) d \bar{x}$.

For $\tilde{\omega}$ to be of the first kind at every point of $\Gamma$, it follows from the first part of the proof that we must have (in addition to the conditions already obtained) that $\phi^{\prime}\left(x^{\prime}, y^{\prime}\right) x^{\prime m-3-h}$ and $\phi(\bar{x}, \bar{y}) \bar{y}^{m-3-h}$ are polynomials in $x^{\prime}, y^{\prime}$ and $\bar{x}, \bar{y}$ respectively, which belong to $\mathfrak{S}_{P}$ for every point $P$ of $\Gamma$ at finite distance in the $\left(X^{\prime}, Y^{\prime}\right)$ - or $(\bar{X}, \bar{Y})$-plane, as the case may be; and conversely. Since $\phi^{\prime}\left(x^{\prime}, y^{\prime}\right)$ and $\bar{\phi}(\bar{x}, \bar{y})$ are not divisible by $x^{\prime}$ or $\bar{y}$ respectively, these conditions imply that $h \leqq m-3$.

There exists then a form $\Phi\left(X_{0}, X_{1}, X_{2}\right)$ of degree $m-3$ such that $\Phi(1, x, y)$ $=\phi(x, y), \Phi\left(x^{\prime}, 1, y^{\prime}\right)=\phi^{\prime}\left(x^{\prime}, y^{\prime}\right) x^{\prime m-3-h}$, and $\Phi(x, y, 1)=\bar{\phi}(\bar{x}, \bar{y}) \bar{y}^{m-3-h}$. It follows that $\tilde{\omega}$ is of the first kind if and only if $\Phi\left(X_{0}, X_{1}, X_{2}\right)=0$ is an adjoint curve to $\Gamma$ of order $m-3$.

The final assertion of the theorem follows directly from the proof. 


\section{BIBLIOGRAPHY}

1. C. Chevalley, On the theory of local rings, Ann. of Math. vol. 44 (1943) pp. 690-708.

2. - Introduction to the theory of algebraic functions of one variable, Mathematical Surveys, no. 6, New York, American Mathematical Society, 1951.

3. W. L. Chow, Die geometrische Theorie der algebraischen Funktionen für beliebige vollkommene Körper, Math. Ann. (1937) pp. 656-682.

4. I. S. Cohen, On the structure and ideal theory of complete local rings, Trans. Amer. Math. Soc. vol. 59 (1946) pp. 54-106.

5. Enriques-Chisini, Lezioni sulla teoria geometrica delle equazioni e delle funzioni algebriche, vol. 2, Bologna, 1915.

6. E. Hecke, Vorlesungen ïber die Theorie der algebraischen Zahlen, Leipzig, Akademische Verlagsgesellschaft, 1923.

7. A. Seidenberg, Valuation ideals in polynomials rings, Trans. Amer. Math. Soc. vol. 57 (1945) pp. 387-425.

8. F. Severi, Trattato di geometria algebrica, vol. 1, part I, Bologna, 1926.

9. — - Vorlesungen über algebraischen Geometrie, Leipzig, 1921.

10. B. L. van der Waerden, Einfïhrung in die algebraische Geometrie, Die Grundlehren der Mathematischen Wissenschaften, Berlin, 1939.

11. O. Zariski, Polynomial ideals defined by infinitely near base points, Amer. J. Math. vol. 60 (1938).

12. - Foundations of a general theory of birational correspondences, Trans. Amer. Math. Soc. vol. 53 (1943) pp. 490-542.

13. - Analytical irreducibility of normal varieties, Ann. of Math. vol. 49 (1948) pp. $352-361$.

Clark University,

WORCESTER, MASS. 